\title{
Rebels without a cause? Zur OMT-Vorlage des Bundesverfassungsgerichts
}

\author{
Von Franz C. Mayer, Bielefeld*
}

Die Euro-Krise und wie sie rechtlich begleitet und bewältigt wird und werden soll ist Gegenstand intensiver Diskussion. Das BVerfG und der EuGH haben sich mit Entscheidungen an dieser Diskussion beteiligt. Mit der Vorlage des BVerfG an den EuGH vom Januar 2014 zum OMT-Programm der EZB, der ersten Vorlage des $B V \operatorname{erfG}$ überhaupt, treten die beiden Gerichte in einen unmittelbaren Austausch zu Fragen der Eurorettung. Der nachfolgende Beitrag analysiert diese erste Vorlage des $B V \operatorname{erf} G$ an den $E u G H$.

„, Wir fahren auf den Abgrund zu. Der erste, der aus dem Wagen springt, der ist der Hasenfuß. “

(Rebel without a cause, 1955/56)

\section{Einleitung}

Eine der berühmtesten Szenen des Filmklassikers „Rebel without a cause“ (1955/56) mit James Dean ${ }^{1}$ ist das sogenannte „Hasenfußrennen“. Zwei Autos rasen auf einen Abgrund zu. Wer als erstes abbremst oder aus dem Auto springt, verliert. Im Film endet das Rennen tragisch: Einer der Protagonisten versucht zu spät, aus dem Wagen zu gelangen und stürzt in den Abgrund. In den jüngeren Entscheidungen des BVerfG zur europäischen Integration und namentlich zur Eurokrise ${ }^{2}$ lassen sich Elemente benennen, die an ein solches Hasenfußrennen erinnern: Ein Gericht bewegt sich immer mehr auf einen Abgrund zu, auf eine ausweglose Situation - immer mehr in einer eigentümlich eigenen Sichtweise und Argumentation befangen, was es ihm zunehmend erschwert, rechtzeitig abzubremsen oder auch nur die Richtung zu ändern.

* Professor, Dr. jur., LL.M. (Yale), Lehrstuhl für Öffentliches Recht, Europarecht, Völkerrecht, Rechtsvergleichung und Rechtspolitik, Universität Bielefeld, Fakultät für Rechtswissenschaften. Kontakt: franz.mayer@uni-bielefeld.de. Ich danke Imke Decker und Marina Ermes (Bielefeld) sowie Dr. Mattias Wendel (Berlin) für zahlreiche Hinweise zu früheren Fassungen dieses Beitrages. Der vorliegende Beitrag beruht auf einem englischsprachigen Text und entwickelt diesen weiter, s. F. C. Mayer, Rebels Without a Cause? A Critical Analysis of the German Constitutional Court's OMT Reference, 15 German Law Journal 2014, S. 111, abrufbar unter http://www.germ anlawjournal.com/.

1 Rebel without a cause, 111 Minuten, Regie Nicholas Ray, Studio Warner Bros., 1955 (USA), 1956 (Deutschland).

2 BVerfG, 2 BvR 2728/13 Beschl. v. 14.1.2014-OMT, http://www.bundesverfassungsgericht.de/entscheidungen/ rs20140114_2bvr272813.html. Daneben hat das BVerfG Aspekte der Eurokrise behandelt in BVerfGE 129, 124 - Griechenland/EFSF; BVerfGE 130, 318 - Neuner-Gremium; BVerfGE 131, 152 - ESM und parlamentarische Kontrolle; BVerfGE 132, 195 - ESM und Fiskalvertrag (einstweiliger Rechtsschutz) sowie BVerfG, 2 BvR 1390/12 u.a. Urt. v. 18.3.2014 - ESM und Fiskalvertrag (Hauptsache) BVerfG, 2 BvR 1390/12, http:// www.bverfg.de/entscheidungen/rs20140318_2bvr139012.html. Ältere Verfahren, die im Hinblick auf den Euro eine Rolle spielen sind BVerfGE 89, 155 - Maastricht; BVerfGE 97, 350 - Euro; BVerfGE 123, 267 - Lissabon. 
Die lange erwartete, nun endlich erfolgte erstmalige Vorlage des BVerfG an den $\mathrm{EuGH}^{3}$ mag man zunächst als ein rechtzeitiges Abbremsen deuten. Bei genauerem Hinsehen kehrt sich dieser Eindruck jedoch um. Die Vorlage belegt zwar die prinzipielle Vorlagebereitschaft des BVerfG, das ist nicht zu beanstanden. Der im Grundsatz zu begrüßende Schritt des BVerfG erfolgt jedoch zur falschen Zeit, im falschen Duktus und am falschen Gegenstand. Jedenfalls ist die Vorlage (dazu II., III.) alles andere als eine Kapitulation, wie es erste Kommentare in den Medien nahelegten (zur Analyse IV.).

\section{Die OMT-Vorlage des BVerfG}

Mit Beschluss vom 14. Januar 2014 hat das BVerfG erstmalig in seiner Geschichte den Gerichtshof der Europäischen Union (EuGH) im Wege des Vorabentscheidungsverfahrens nach Art. 267 AEUV angerufen. Gegenstand der Vorlage ist das im September 2012 durch den Präsidenten der Europäischen Zentralbank (EZB) Draghi angekündigte OMT-Programm ${ }^{4}$ der EZB und seine Vereinbarkeit mit Unionsrecht. Fragen um das OMT-Programm waren zunächst Teil des Verfahrens um die Rechtmäßigkeit des ESM-Vertrages und des Fiskalvertrages. In diesem Verfahren lehnte das BVerfG im September 2012 Anträge auf Eilrechtsschutz $a b^{5}$ und machte damit den Weg frei für die deutsche Ratifikation der beiden Vertragswerke. Dabei spielte das OMT-Programm allerdings noch keine Rolle, da es erst eine Woche zuvor bekannt geworden war. In der mündlichen Verhandlung zur Hauptsache im Juni 2013 stand das OMT-Programm dagegen im Vordergrund. Die Entscheidung, die OMT-Aspekte von dem den ESM/Fiskalvertrag betreffenden Teil des Verfahrens abzutrennen, erging im Dezember 2013. ${ }^{6}$ Der Vorlagebeschluss des Zweiten Senates vom Januar 2014 erging mit 6 zu 2 Stimmen. Die beiden dienstältesten Richter des Zweiten Senats, Gertrude Lübbe-Wolff und Michael Gerhardt, gaben Sondervoten ab.

3 Die Rs. ist beim EuGH unter dem Aktenzeichen C-62/14, Gauweiler u.a., anhängig. Peter Gauweiler ist Mitglied des Deutschen Bundestages und stellvertretender Vorsitzender der CSU, er war bereits Beschwerdeführer im Lissabon-Verfahren vor dem BVerfG und kann als Europaskeptiker gelten. Andere Beteiligte sind in diesem Verfahren u.a. eine Nichtregierungsorganisation zur Förderung der direkten Demokratie und die Fraktion DIE LINKE im Deutschen Bundestag.

4 Beschluss des Rates der Europäiischen Zentralbank v. 6.9.2012 betreffend Outright Monetary Transactions (OMT). Ausweislich der Sachverhaltsdarstellung in einer gegen das OMT-Programm vor dem europäischen Gericht erster Instanz angestrengten erfolglosen Klage ist der Text der fraglichen Beschlüsse lediglich in EZBPressemitteilungen v. 6.9.2012 wiedergegeben. Danach gibt es keinen gesonderten Text, in dem diese Beschlüsse förmlich festgehalten worden sind, EuG, Rs. T-492/12 (von Storch u.a./EZB), Beschl. v. 10.12.2013, Rn. 2.

5 S.o. Fn. 2, näher dazu $M$. Wendel, Judicial Restraint and the Return to Openness: The Decision of the German Federal Constitutional Court on the ESM and the Fiscal Treaty of 12 September 2012, 14 German Law Journal 2013, S. 21.

6 BVerfG, 2 BvR 1390/12, Beschl. v. 17.12.2013, abrufbar unter: http://www.bverfg.de/entscheidungen/rs20140 114_2bvr272813.html. Die endgültige Entscheidung zu dem den ESM/Fiskalvertrag betreffenden Teil des Verfahrens erging übrigens am 18.3.2014 und bestätigte im Wesentlichen die Linie aus der Eilrechtsschutzentscheidung, BVerfG, 2 BvR 1390/12, Urt. v. 18.3.2014, http://www.bverfg.de/entscheidungen/rs20140318_2bvr 139012.html. 
Die Nichtvorlagepraxis des BVerfG war bis zur Vorlage vom Januar 2014 Gegenstand einer anhaltenden Diskussion bis hin zu Vorschlägen einer einfachgesetzlichen Verankerung der bundesverfassungsgerichtlichen Vorlagepflicht. ${ }^{7}$ Nicht selten heißt es, die Vorlage eines Verfassungsgerichts sei wegen der unterschiedlichen Jurisdiktionsbereiche zwischen Verfassungsgericht und EuGH, Verfassungsrecht gegenüber Europarecht, kein Thema. ${ }^{8}$ In Deutschland wurde allerdings zunehmend deutlicher, dass eine Vorlage des BVerfG eigentlich nur noch eine Frage der Zeit war. ${ }^{9}$ Mit der nun endlich erfolgten Vorlage löst das BVerfG diese Erwartung ein. Gleichwohl bleibt der Applaus bisher aus. ${ }^{10}$ Die Reaktionen ${ }^{11}$ im In- und Ausland reichen von der Bewertung des Vorlagebeschlusses als verfassungswidrig ${ }^{12}$ bis zu der Diagnose, die Senatsmehrheit befinde sich in einer anderen Galaxie und dem Vorwurf der richterlichen Arroganz. ${ }^{13}$

7 J. Bergmann/U. Karpenstein, Identitäts- und Ultra-vires-Kontrolle durch das BVerfG - zur Notwendigkeit einer gesetzlichen Vorlageverpflichtung, ZEuS 2009, S. 529. Möglicherweise wäre auch nach der OMT-Vorlage eine einfachgesetzliche Regelung im BVerfGG sinnvoll. Darin könnten prozedurale Aspekte einer Vorlage des BVerfG näher ausgestaltet werden. Dies betrifft Fragen wie die der mündlichen Verhandlung vor oder nach einer Vorlage, Sondervoten zum Vorlagebeschluss, die Beteiligung von Verfassungsorganen am Vorlageverfahren in Luxemburg. S. im Übrigen die Nachweise zur älteren Diskussion und zur Vorlagepraxis von Höchstgerichten in F. C. Mayer, Das BVerfG und die Verpflichtung zur Vorlage an den EuGH, EuR 2002, S. 239.

8 Symptomatisch hier das ungarische Verfassungsgericht Alkotmánybíróság in einer seiner allerersten Entscheidungen mit EU-Bezug, Entscheidung 17/2004 (V.25) AB v. 25.5.2004, die ein EU-Vorgaben umsetzendes Gesetz für verfassungswidrig erklärte: Gegenstand seiner Entscheidung sei allein die Verfassungsmäßigkeit ungarischen Rechts, nicht dagegen die Vereinbarkeit mit Unionsrecht.

9 S. bereits BVerfGE 37, 271, 282 - Solange I; aus jüngerer Zeit etwa BVerfGE 110, 141, (154 f.) - Kampfhunde, Rn. 57.

10 Krit. etwa I. Pernice, A Difficult Partnership between Courts: The First Preliminary Reference by the German Federal Constitutional Court to the CJEU, Maastricht Journal of European and Comparative Law 2014, S. 3.

11 Vgl. etwa M. Ruffert, Vorlagebeschluss des BVerfG zum OMT-Programm, JuS 2014, S. 373; C. Hillgruber, Anmerkung zu BVerfG: Die verfassungsprozessuale Dimension des Outright Monetary Transactions (OMT)Beschluss des Bundesverfassungsgerichts, JA 2014, S. 635; D. Baumann, Anmerkung zu BVerfG: Vereinbarkeit des Ankaufs von Staatsanleihen durch die EZB mit Unionsrecht, GWR 2014, S. 199; R. Brosius-Linke, Die Vorlageentscheidung des Bundesverfassungsgerichts, DÖV 2014, S. 612; S. Müller-Franken, Zur Unionsrechtskonformität des OMT-Beschlusses, NVwZ 2014, S. 514; W. Frenz, Zur Vorabentscheidungsvorlage hinsichtlich des OMT-Beschlusses der EZB, DVB1. 2014, S. 451; E. R. Zivier, Konzert oder Duell? Zum Vorlagebeschluss des Bundesverfassungsgerichts an den Europäischen Gerichtshof vom 14. Januar 2014, Recht und Politik 2014, S. 85, J. H. Klement, Der Euro und seine Demokratie. Zu den Entscheidungen des Bundesverfassungsgerichts vom 14.01. und 18.03.2014 in Sachen "Outright Monetary Transactions" und Europäischer Stabilitätsmechanismus, Zeitschrift für Gesetzgebung 2014, S. 169; C. D. Classen, Alle Macht den Richtern?, jM 2014, S. 345; C. Herrmann, Luxemburg, wir haben ein Problem!, EuZW 2014, S. 161; M. Wendel, Exceeding Judicial Competence in the Name of Democracy: The German Federal Constitutional Court's OMT Reference, European Constitutional Law Review 2014, S. 263; ders., Kompetenzrechtliche Grenzgänge, ZaöRV 2014, i.E.; S. Dahan, O. Fuchs, M.-L. Layus, ,,Whatever it takes ?": à propos de la décision OMT de la Cour constitutionnelle fédérale d'Allemagne, AJDA 2014, S. 1311. S.auch die 14 Beiträge im Special Issue OMT, Issue 2 German Law Journal Volume 15 (2014), http://www.germanlawjournal.com/.

12 W. Heun, Eine verfassungswidrige Verfassungsgerichtsentscheidung - der Vorlagebeschluss des BVerfG vom 14.1.2014, JZ 2014, S. 331.

13 „Germany's constitutional judges seem to live in a far, far away galaxy from the financial reality.", Ch. Secondat, P.J. Goossens, D. Roterod, The German Constitutional Court's decision about the European Central Bank's OMT mechanism: A masterpiece of judicial arrogance, European Policy Brief v. 30.4.2014. 


\section{Oft gestellte Fragen (FAQs)}

Das OMT-Programm berührt eine Vielzahl von komplexen faktischen, ökonomischen und rechtlichen Zusammenhängen. Es empfiehlt sich, zunächst eine Reihe von oft gestellten Fragen abzuschichten.

\section{Worum geht es eigentlich?}

Staatsaufgaben kosten Geld. Neben Steuern kommen als Einnahmequelle zur Deckung der entsprechenden Ausgaben Staatsanleihen in Betracht. Staaten leihen sich dabei gegen einen bestimmten Zinssatz Geld am Kapitalmarkt. Der Zinssatz reflektiert den Grad des Vertrauens der Investoren in die Wahrscheinlichkeit, dass sie ihr Geld zurückbekommen. Steigt der Zinssatz über einen bestimmten Schwellenwert, geraten Staaten in Liquiditätsprobleme - es geht ihnen das Geld aus. In der Eurokrise war die Lösung für dieses Problem die Einführung des Europäischen Stabilitätsmechanismus (ESM). Der ESM kann über Kredite Geld und andere Finanzhilfen für Staaten in Schwierigkeiten bereitstellen, dies allerdings nur unter strengen Bedingungen (,Konditionalitäten“) für die um Unterstützung bittenden Staaten. ${ }^{14}$ Der ESM beschafft sich seinerseits Mittel hauptsächlich am Finanzmarkt. ${ }^{15}$

Im September 2012 erlaubte das BVerfG im Eilrechtsschutzverfahren ${ }^{16}$ die deutsche Beteiligung am ESM unter der Voraussetzung, dass der Bundestag jederzeit die Kontrolle über Maßnahmen des ESM behält. Das bedeutet, dass der deutsche Regierungsvertreter in den beschlussfassenden Organen des ESM die vorherige Erlaubnis des Bundestags benötigt, bevor er seine Zustimmung zu einer Maßnahme des ESM erteilt. ${ }^{17}$

In einer parallelen Entwicklung wurde ebenfalls im September 2012 bekannt, dass die EZB falls notwendig Staatsanleihen von in Schwierigkeiten geratenen Eurostaaten auf dem Sekundärmarkt aufkaufen würde, um den Druck auf diese Staaten zu mindern und das Funktionieren der Währungsunion zu sichern. Bedingung soll dabei sein, dass sich diese Staaten zur Teilnahme an einem ESM-Programm ${ }^{18}$ und damit zur Einhaltung strenger Bedingungen, in der Regel Reform- und Sparmaß-

14 S. für Einzelheiten http://www.esm.europa.eu/. Der ESM ersetzt den zeitlich begrenzten EFSF.

15 Es besteht allerdings ein Kapitalstock.

16 Bestätigt in BVerfG, 2 BvR 1390/12 u.a. Urt. v. 18.3.2014 - ESM und Fiskalvertrag (Hauptsache) BVerfG, 2 BvR 1390/12, http://www.bverfg.de/entscheidungen/rs20140318_2bvr139012.html. Dazu C. Tomuschat, Anmerkung, DVB1. 2014, S. 645 (646).

17 S. für Einzelheiten das Gesetz zur finanziellen Beteiligung am Europäischen Stabilitätsmechanismus, (ESMFinanzierungsgesetz, ESMFinG). In den Worten des BVerfG: „Aus der demokratischen Verankerung der Haushaltsautonomie folgt jedoch, dass der Bundestag einem intergouvernemental oder supranational vereinbarten, nicht an strikte Vorgaben gebundenen und in seinen Auswirkungen nicht begrenzten Bürgschafts- oder Leistungsautomatismus nicht zustimmen darf, der - einmal in Gang gesetzt - seiner Kontrolle und Einwirkung entzogen ist. (BVerfGE 129, 124, 180).“, BVerfGE 132, 195, 241 - ESM und Fiskalvertrag (einstweiliger Rechtsschutz).

18 Genannt wird auch der Vorläufer zum ESM, EFSF. 
nahmen, verpflichten. Niedergelegt ist dies in der Entscheidung des EZB-Rats vom 6. September 2012 betreffend „Technical Features of Outright Monetary Transactions“ (OMT). ${ }^{19}$ Bereits die bloße Pressemitteilung mit der das OMT-Programm lediglich angekündigt wurde reichte aus, um die Märkte zu beruhigen. Eine Aktivierung des Programms hat in der Folge nicht stattgefunden. Dem Anschein nach verfolgt das OMT-Programm denselben Zweck wie der ESM, nämlich die Liquidität von in Schwierigkeiten geratenen Staaten zu sichern - offensichtlich aber ohne Beteiligung des Bundestages. Es ist unschwer nachzuvollziehen, dass man im BVerfG nicht begeistert davon war, dass die EZB das ausgeklügelte Konstrukt des BVerfG zur Sicherung weitreichender ESM-Einwirkungsrechte für den Bundestag zum Schutze des deutschen Steuerzahlers durch eine dürre Pressemitteilung überspielen konnte. Maßnahmen der EZB erfordern weder die Zustimmung eines nationalen Verfassungsgerichts noch die eines mitgliedstaatlichen Parlaments. ${ }^{20}$

Die Beschwerdeführer und Antragsteller der gegen den ESM und den Fiskalpakt anhängigen Verfassungsbeschwerden und Organklagen, in denen das BVerfG im September 2012 Eilrechtsschutz abgelehnt hatte, brachten die OMT-Frage in der Folge in das Verfahren ein. Die mündliche Verhandlung im Hauptverfahren im Juni 2013 stellte sich in weiten Teilen als ein Tribunal über ökonomische Aspekte des OMT-Programms der EZB dar. ${ }^{21}$

Es ist diese Konfrontation zwischen BVerfG und EZB - beides übrigens Institutionen, die keiner unmittelbaren demokratischen Kontrolle unterworfen sind - die an das Hasenfußrennen aus „Rebel without a cause“ (s.o.) erinnert.

\section{Ist das OMT-Programm der EZB mit Europarecht vereinbar?}

Nach Auffassung der Senatsmehrheit: Nein. ${ }^{22}$ Die Richter meinen, das OMT-Programm sei nicht mehr vom Mandat der EZB umfasst und nicht mehr Geldpolitik. ${ }^{23}$ Sie sehen auch das Verbot der Staatsfinanzierung verletzt. Die Senatsmehrheit argumentiert, dass die Abgrenzung zwischen Wirtschaftspolitik und Geldpolitik sich nach dem objektiv zu bestimmenden Ziel einer Maßnahme richte und sie weisen auf die Argumente des EuGH in Bezug auf die Grenzziehung zwischen Kompetenzen der Mitgliedstaaten und solchen der EU in der Rs. Pringle hin. ${ }^{24}$ Dabei un-

19 Verfügbar unter http://www.ecb.europa.eu/. Einzelheiten der EZB-Maßnahme sind auch erklärt in dem Beschl. des EuG v. 10.12.2013 zum OMT-Programm, Rs. T-492/12 (von Storch u.a./EZB), Rechtsmittel anhängig beim EuGH als Rs. C-64/14 P. Das OMT-Programm ist Nachfolger des Securities Market Programme (SMP, ab Mai 2010 bis September 2012), das ebenfalls darauf abzielte, Anleihen von in Schwierigkeiten geratenen EuroMitgliedstaaten am Sekundärmarkt zu kaufen.

20 Die Senatsmehrheit macht sehr deutlich, dass sie ESM und OMT in einer funktionalen Parallele sieht, BVerfG, 2 BvR 2728/13 Beschl. v. 14.1.2014 - OMT, Rn. 40 und 78.

21 Beobachter hielten die Auswahl an Ökonomen, die vom Gericht als sachkundige Dritte angehört wurden, für auffällig EZB-kritisch, s. dazu die Analyse der Positionen in der mündlichen Verhandlung zu OMT unter: http:// www.bruegel.org/nc/blog/detail/article/1109-overview-of-the-karlsruhe-hearing-on-omt-summary/.

22 BVerfG, 2 BvR 2728/13, Beschl. v. 14.1.2014-OMT, Rn. 55-98.

23 BVerfG, 2 BvR 2728/13, Beschl. v. 14.1.2014 - OMT, Rn. 63, 69.

24 EuGH, Rs. C-370/12 (Pringle/Irland), Slg. 2012, I-00000. 
terstellt die Senatsmehrheit freilich von vornherein, dass das OMT-Programm und der ESM gleichsam artgleiche austauschbare Instrumente zur Bewältigung der Euro-Krise sind ${ }^{25}$ - was so nicht stimmt - und dass es zwei hermetisch getrennte Welten gibt, die der Wirtschaftspolitik und die der Währungspolitik. Zwar betont auch der EuGH in Pringle, dass es mittelbare Effekte zwischen verschiedenen Maßnahmen geben kann. ${ }^{26}$ Aus dem Pringle-Urteil lassen sich indes keine Schlussfolgerungen für die Rechtmäßigkeit des OMT-Programms ableiten, weil es um einen anderen Gegenstand geht. ${ }^{27}$

Ferner verweist die Senatsmehrheit im Vorlagebeschluss des BVerfG auf,,die überzeugende Expertise der Bundesbank". ${ }^{28}$ Hier bleibt freilich offen, warum diese Expertise mehr überzeugt als die zahlreicher Wirtschaftswissenschaftler in aller Welt, ${ }^{29}$ die freilich in der mündlichen Verhandlung unterrepräsentiert waren. ${ }^{30}$ Vieles spricht dafür, dass es in gewissem Sinne gar keine überzeugende Expertise in die eine oder andere Richtung gibt und das Thema fachlich umstritten und damit auch rechtlich - nicht auflösbar bleibt.

Die Vorlagefragen des BVerfG beziehen sich auf die Auslegung der maßgeblichen Bestimmungen des Unionsrechts, ${ }^{31}$ legen aber zugleich die Antwort bereits nahe. Allenfalls durch eine enge Auslegung des Unionsrechts, so die Senatsmehrheit, könne das OMT-Programm sich noch mit Unionsrecht vereinbaren lassen. Diese enge Auslegung richtet sich insbesondere, aber nicht nur auf Grenzen bezüglich des Gesamtumfangs der Sekundärmarktankäufe. Die sechs Richter der Senatsmehrheit machen dem EuGH in Randnummer 100 der Entscheidung im Einzelnen recht detaillierte Vorgaben (siehe unten für Einzelheiten). Das Problem ist dabei, dass das OMT-Programm mit all diesen Einschränkungen seinen Charakter verlieren und wahrscheinlich wirkungslos würde.

Weitaus überzeugender als die Senatsmehrheit erscheinen die beiden Sondervoten. Bedauerlich ist dabei allenfalls, dass die beiden dienstältesten Richter nicht bereits in früheren Verfahren das Wort ergriffen haben, um einige Dinge klar auszusprechen. Hier wird betont, dass die Darstellung der EZB, das OMT-Programm sei Geldpolitik mit dem vorrangigen Ziel der Wiederherstellung des monetären Transmissionsmechanismus, nicht entkräftet werden könne. ${ }^{32}$ Dies läuft auf eine Zuweisung der fraglichen Themen in den Bereich der politischen Fragen hinaus, bei denen das Gericht gut beraten ist, Abstand zu halten. Dieser Ansatz verdient Zustimmung.

25 S. dazu oben Fn. 20.

26 EuGH, Rs. C-370/12 (Pringle/Irland), Slg. 2012, I-00000 Rn. 56.

27 Näher dazu M. Wendel, Kompetenzrechtliche Grenzgänge, ZaöRV 2014, i.E.

28 BVerfG, 2 BvR 2728/13, Beschl. v. 14.1.2014 - OMT, Rn. 71.

29 S. dazu den Aufruf zur Unterstützung des Anleihekaufprogramms OMT der Europäischen Zentralbank v. 19.7.2013, http://berlinoeconomicus.diw.de/geldpolitik/. Dem stellten sich deutsche Nationalökonomen entgegen, Deutsche Ökonomen werfen der EZB Staatsfinanzierung vor, FAZ v. 11.9.2013.

30 S. dazu Fn. 21.

31 Die maßgeblichen Vorschriften sind Art. 119 und 127 Abs. 1 und 2 AEUV und Art. 17 bis 24 des Protokolls über die Satzung des Europäischen Systems der Zentralbanken und der Europäischen Zentralbank; Art. 123 AEUV und 130 AEUV spielen ebenfalls eine Rolle.

32 BVerfG, 2 BvR 2728/13, Beschl. v. 14.1.2014 - OMT, Sondervotum Gerhardt, Rn. 7. 
Es bleibt rätselhaft, wie die sechs Richter der Senatsmehrheit zu der Überzeugung gelangen konnten, dass sie eine Frage zu entscheiden vermögen, die bereits unter Ökonomen heftig umstritten war und nach wie vor ist. ${ }^{33}$ Wie im Sondervotum Lübbe-Wolff dargelegt, ${ }^{34}$ erscheint weniger die EZB als vielmehr das BVerfG als die Institution, die Schranken ihrer Befugnisse und ihrer Sachkenntnis überschreitet.

\section{Warum kann ein mitgliedstaatliches Gericht eigentlich eine europäische Maßnahme überprüfen und sogar für ungültig erklären?}

Dass das BVerfG die Rechtmäßigkeit des OMT-Programms der EZB überhaupt überprüfen kann, verblüfft. Die Auslegung von Unionsrecht erscheint intuitiv nicht gerade als eine naheliegende Aufgabe für mitgliedstaatliche Verfassungsgerichte. Tatsächlich stellen sich in den Mitgliedstaaten tagtäglich Auslegungsfragen des Unionsrechts in nationalen Verfahren aller Instanzen. Im Verwaltungsverfahren besteht für die nationalen Behörden keine Möglichkeit, die richtige Auslegung des Unionsrechts zu erfragen. Anders bei den Gerichten: Um die einheitliche Anwendung von Unionsrecht zu sichern, können bzw. müssen nationale Gerichte den EuGH im Wege des Vorabentscheidungsverfahrens befassen, soweit eine Auslegungs- oder Gültigkeitsfrage des Unionsrechts für den nationalen Fall entscheidungserheblich ist (Art. 267 AEUV). Auf der Grundlage der Auslegung des Unionsrechts durch ein Urteil des EuGH im Vorabentscheidungsverfahren ergeht dann auf nationaler Ebene durch das nationale Gericht das Endurteil.

In ständiger Rechtsprechung gestattet der EuGH es den nationalen Gerichten grundsätzlich nicht, Unionsrecht für ungültig oder nicht anwendbar zu erklären. ${ }^{35}$ Nur so kann die einheitliche Anwendung des Unionsrechts gesichert und die Entwicklung von potentiell 29 unterschiedlichen Versionen des Unionsrechts - 28 Versionen der Mitgliedstaaten und eine zusätzliche des EuGH - unterbunden werden. Andernfalls droht ein erhebliches Maß an Rechtsunsicherheit. Die Verträge sehen eindeutig und ausschließlich den EuGH als letzte Instanz für die Auslegung des Unionsrechts vor. In Art. 344 AEUV heißt es wörtlich: „Die Mitgliedstaaten verpflichten sich, Streitigkeiten über die Auslegung oder Anwendung der Verträge nicht anders als hierin vorgesehen zu regeln."

Das BVerfG hat dies jedoch bisher nicht vollständig akzeptiert. Die anfänglich im Vordergrund stehenden Vorbehalte des BVerfG bei Grundrechtsfragen können dabei heute als minimiert angesehen werden. Das BVerfG hat im Laufe der Zeit eine Formel gefunden, die es ihm zwar theoretisch noch immer ermöglicht, Akte der EU im Hinblick auf Grundrechtsschutz zu überprüfen. Zugleich gesteht das BVerfG indessen dem EuGH den Vorrang zu, wenn es um Fragen des Grundrechtsschutzes

\footnotetext{
S. dazu Fn. 29.

BVerfG, 2 BvR 2728/13, Beschl. v. 14.1.2014 - OMT, Sondervotum Lübbe-Wolff, Rn. 3.

5 EuGH, Rs. 314/85 (Foto-Frost/Hauptzollamt Lübeck-Ost), Slg. 1987, 4199, 4231.
} 
gegen Maßnahmen der EU geht und übt die von ihm beanspruchte Rechtsprechungskompetenz in Grundrechtsfragen bis auf weiteres nicht mehr aus. ${ }^{36}$

Deutlicher ist der Gegensatz zwischen EuGH und BVerfG in der Frage der Kompetenzkontrolle. Zwar hatte auch hier das BVerfG die Zuständigkeit des EuGH bereits anerkannt. In der Vielleicht-Entscheidung aus dem Jahre 1979 heißt es wörtlich:

„Art. 177 EWGV [heute Art. 267 AEUV] spricht dem Gerichtshof im Verhältnis zu den Gerichten der Mitgliedstaaten die abschließende Entscheidungsbefugnis über die Auslegung des Vertrages sowie über die Gültigkeit und die Auslegung der dort genannten abgeleiteten gemeinschaftsrechtlichen Akte zu. "37

Mit dem Maastricht-Urteil von $1993^{38}$ vollzog das BVerfG dann aber eine Abkehr von dieser Anerkennung der europavertragsrechtlichen Bindungen der Bundesrepublik (s.o., Art. 344 AEUV) und formulierte eine Ultra vires-Doktrin mit einem Kontrollvorbehalt über die Kompetenzausübung der EU. Demzufolge soll das BVerfG prüfen können, ob Maßnahmen der europäischen Einrichtungen und Organe mit den für die Übertragung von Hoheitsrechten auf die EU aufgestellten Grenzen übereinstimmen. ${ }^{39}$ Das Gericht rechtfertigt seine Kontrollbefugnis über Ultra vires-Akte (in der Entscheidung von 1993 spricht das BVerfG noch von ausbrechenden Rechtsakten ${ }^{40}$ ) aus seiner Zuständigkeit für die Integrationsschranken des Grundgesetzes. Dem BVerfG zufolge kann das Integrationsprogramm, ${ }^{41}$ das vom Zustimmungsgesetz und den Gründungsverträgen umrissen wird, nicht im Nachhinein im Wege europäischer Ultra vires-Akte substanziell geändert werden, ohne die Deckung durch das Zustimmungsgesetz zu verlieren.

Dem Anschein nach geht es dabei lediglich um deutsches Recht, nämlich das Zustimmungsgesetz zum jeweiligen Primärrecht. Tatsächlich läuft das Konzept des ausbrechenden Rechtsakts auf eine autonome bundesverfassungsgerichtliche Auslegung des Unionsrechts hinaus. Das BVerfG überprüft eine bestimmte Maßnahme der EU auf ihre Vereinbarkeit mit dem deutschen Zustimmungsgesetz zum jeweils einschlägigen Primärrecht. Mit der Frage nach dem Einhalten oder Überschreiten des Integrationsprogramms durch europäische Einrichtungen und Organe verbindet sich jedoch zwangsläufig der Blick hinüber in die Unionsrechtsordnung, mit allen

36 BVerfGE 37, 271, 282 - Solange I; BVerfGE 73, 339 - Solange II; BVerfGE 102, 147 - Bananenmarkt.

37 BVerfGE 52, 187, 202 - Vielleicht.

38 BVerfGE 89, 155 - Maastricht; die Entscheidung und die Verfahren sind gut dokumentiert bei I. Winkelmann, Das Maastricht-Urteil des BVerfGs vom 12. Oktober 1993 (1994); weitere Nachweise bei F. C. Mayer, Kompetenzüberschreitung und Letztentscheidung, 2000, S. 98-116 und bei ders., Verfassungsgerichtsbarkeit, in: v. Bogdandy/Bast (Hrsg.), Europäisches Verfassungsrecht, 2009, S. 399 ff.

39 BVerfGE 89, 155, 188 - Maastricht.

40 Für den Begriff s. schon die frühere Entscheidung des BVerfG BVerfGE 75, 223, 242 - Kloppenburg. Für die Unterscheidung zwischen Ultra vires-Akten in einem engeren Sinne (Überschreiten bestimmter Kompetenzen in Bezug auf ein Sachgebiet) und in einem weiteren Sinne (generelle Rechtswidrigkeit einer Maßnahme) $F$. $C$. Mayer, Kompetenzüberschreitung (Fn. 38), S. 24 ff.

41 Eigenartigerweise benutzt das BVerfG die Idee eines einem Vertrag zugrundeliegenden „Integrationsprogramms" auch im Kontext der NATO, BVerfGE 104, 151 - Neues Strategisches Konzept der NATO; s. dazu M. Rau, NATO's New Strategic Concept, 44 GYIL 2001, S. 545, 570. 
Risiken der Fehleinschätzung, die der fachfremde, methodisch, sprachlich und kulturell außerhalb der eigenen vertrauten fachgerichtlichen Bahnen unternommene Interpretationsversuch birgt. Akte der EU werden letztlich am Maßstab einer deutschen Version des Unionsrechts überprüft. Die vermeintliche Beschränkung des Prüfungsgegenstandes auf eine Überprüfung des Zustimmungsgesetzes erweist sich damit letztlich als Trick: Tatsächlich hängt die Vereinbarkeit einer europäischen Maßnahme mit deutschem Verfassungsrecht von ihrer Vereinbarkeit mit Unionsrecht (in der bundesverfassungsgerichtlichen Variante), nämlich ihrer Kompetenzkonformität, ab. Der Sache nach erfolgt eine Verdopplung des Kontrollmaßstabes. Unionsakte müssen mit den Garantien des Grundgesetzes und mit Unionsrecht vereinbar sein.

Rechtsfolge der Feststellung, dass eine europäische Maßnahme sich als ultra vires - kompetenzwidrig - erweist, ist der Verlust der Verbindlichkeit des betreffenden Rechtsaktes in Deutschland. Im Geltungsbereich des Grundgesetzes sind Ultra vires-Akte schlicht unbeachtlich. Ob man dem „Rechtsanwendungsbefehl“ des Unionsrechts in einem solchen Falle gewissermaßen freiwillig nachkommen darf, hängt dann davon ab, ob im Übrigen ein Verstoß gegen das Grundgesetz besteht. Es ergibt sich insgesamt ein aus deutschem Verfassungsrecht begründeter Kontrollvorbehalt über europäische Akte, der letztlich den Vorranganspruch des Europarechts einschränkt. Da es niemals eine Kompetenz zur Setzung rechtswidrigen Rechts gibt und der europäischen Hoheitsgewalt mit Sicherheit keine solche Rechtsmacht für rechtswidriges Recht übertragen worden ist, ist der Ultra vires-Vorbehalt potenziell ein allgemeiner Rechtmäßigkeitsvorbehalt. Das BVerfG beansprucht das letzte Wort über die Rechtmäßigkeit von Unionsrecht und damit die Rolle als letzte Instanz in Fragen des Unionsrechts in Deutschland.

\section{Warum haben die sechs Richter der Senatsmehrheit dem EuGH überhaupt vorgelegt, obwohl sie doch offenbar überzeugt davon sind, dass das OMT- Programm nicht vom Mandat der EZB gedeckt ist?}

Einige Beobachter ${ }^{42}$ haben eine schlichte Antwort auf diese Frage: Demnach gab es im Senat drei Fraktionen. Neben den beiden abweichenden Richtern gab es offenbar Richter, die das OMT-Programm ohne weiteres gleich für ultra vires erklären wollten, daneben Richter, die darauf bestanden, zunächst dem EuGH vorzulegen. Dann wäre der Vorlagebeschluss schlicht der Kompromiss zwischen den beiden letztgenannten Fraktionen. Diese Deutung erklärt, warum eine an sich ergebnisoffene Vorlagefrage hier bereits im Duktus einer Feststellung, nämlich einer Ultra vires-Feststellung, daherkommt.

Die etwas differenziertere Antwort lautet wie folgt: Das BVerfG hat seine Ultra vires-Doktrin im Laufe der Zeit weiterentwickelt. Ohne seit der Erfindung dieser 
Doktrin mit dem Maastricht-Urteil 1993 jemals eine europäische Maßnahme für ultra vires erklärt haben zu müssen, hat das Gericht im Lissabon-Urteil ${ }^{43} 2009$ die Ultra vires-Doktrin bestätigt und zudem gegenüber dem Gesetzgeber angeregt, die Ultra vires-Kontrolle sogar gesetzlich zu verankern, etwa durch eine Änderung des BVerfGG, mithin das richterrechtliche Konstrukt zu kodifizieren. Der Gesetzgeber hat dieses Ansinnen ignoriert.

Wohl nicht zuletzt als Reaktion auf die überwiegende Negativkritik an der LissabonEntscheidung ${ }^{44}$ hat das BVerfG in seiner Honeywell-Entscheidung 2010 seine Ultra vires-Doktrin neu formuliert. ${ }^{45}$ Dabei wurden die Voraussetzungen für eine Ultra vires-Entscheidung, die Unionsrecht für in Deutschland unbeachtlich erklärt, derart streng gefasst, dass es nach Honeywell nahezu hypothetisch erscheinen musste, dass das BVerfG tatsächlich jemals Unionsrecht für ultra vires erklären würde: Neben dem grundsätzlichen Erfordernis, die Ultra vires-Kontrolle europarechtsfreundlich auszuüben, ${ }^{46}$ muss die in Rede stehende Maßnahme Kompetenzen der EU evident überschreiten, es muss dadurch zu einer strukturellen Verschiebung im Kompetenzgefüge zwischen der EU und den Mitgliedstaaten kommen, und der EuGH muss die Möglichkeit gehabt haben, die angegriffene Maßnahme der EU zu überprüfen. Dies impliziert, dass, falls noch keine Vorlage erfolgte, jedenfalls das BVerfG den EuGH anruft, wenn es beabsichtigt, eine Maßnahme der EU für ultra vires zu erklären.

Genau dies ist der Sachstand in Sachen OMT: Die Senatsmehrheit hält das OMTProgramm für eine evidente Ultra vires-Handlung, die strukturell das Kompetenzgefüge zwischen EU und Mitgliedstaaten verändert. Danach wird schlicht die Honeywell-Checkliste abgearbeitet. Auf die Einordnung einer Maßnahme als kompetenzwidrig folgt, dass sicherzustellen ist, dass der EuGH die Möglichkeit hatte, die streitige Maßnahme seinerseits für kompetenzwidrig und rechtswidrig zu erklären, wodurch weitere Schritte des BVerfG überflüssig würden.

\section{Wird der EuGH eine Frage überhaupt beantworten, die eher einem Diktat als einer Frage gleicht?}

Es ist aus verschiedenen Gründen ungewiss, ob die vom BVerfG vorgelegte Frage den formellen Anforderungen des Vorabentscheidungsverfahrens nach Art. 267 AEUV (Zulässigkeit der Vorlagefrage) genügt. Ob der Beschluss der EZB und die ihr mögliches zukünftiges Vorgehen betreffende Pressemitteilung - im Grunde das einzige, was vom OMT-Programm bisher sichtbar ist - bereits Rechtswirkungen

43 BVerfGE 123, 267, 253 ff. - Lissabon.

44 S. zum Beispiel die Beiträge von Ch. Schönberger, Ch. Möllers/D. Halberstam und Ch. Tomuschat in der Sonderausgabe zum Lissabon-Urteil des BVerfG, 10 German Law Journal 2009, Issue 8.

45 BVerfGE 126, 286, 303-307 - Honeywell. S. dazu im Einzelnen F. C. Mayer/M. Walter, Die Europarechtsfreundlichkeit des BVerfG nach dem Honeywell-Beschluss, Jura 2011, S. 532.

46 BVerfGE 126, 286, 303 - Honeywell. 
entfaltet, erscheint zweifelhaft. ${ }^{47}$ Das OMT-Programm wurde noch gar nicht initiiert, und wird es möglicherweise auch nie werden. Es wäre für den EuGH ein 1Leichtes, die Vorlage als unzulässig zu verwerfen und dem BVerfG zu bedeuten, dass es seine Frage im Vorabentscheidungsverfahren dann erneut vorlegen kann, wenn das OMT-Programm auch rechtlich konkreter wird. Gegenwärtig ist OMT lediglich eine unbestimmte Ankündigung möglicher Handlungen der EZB in der unbestimmten Zukunft.

Das BVerfG antizipiert dieses Problem ${ }^{48}$ und legt dem EuGH einen alternativen Fragenkatalog vor, der nicht auf den Beschluss und die Pressemitteilung der EZB zum OMT-Programm abstellt, sondern allgemein auf eine Entscheidung des „Eurosystems". Damit ist indessen nichts gewonnen, weil der EuGH im Rahmen des Vorabentscheidungsverfahrens im Grundsatz keine hypothetischen Fragen akzeptiert. Der EuGH formuliert hierzu in ständiger Rechtsprechung, ,dass Artikel [267 AEUV] dem Gerichtshof nicht die Aufgabe zuweist, Gutachten zu allgemeinen oder hypothetischen Fragen abzugeben." 49

Das im Vorabentscheidungsverfahren vorlegende nationale Gericht muss vielmehr eine echte europarechtliche Frage, die für einen realen Ausgangsfall entscheidungserheblich ist, vorweisen können. Eines der Sondervoten weist hier auf ein weiteres Problem hin: Stellt es eigentlich noch eine Frage dar, wenn man bereits unterstellt, dass die eigenen Erwägungen doch die richtige Antwort sind und dies entsprechend - wie hier - äußert ${ }^{50}$ Hier ist zu differenzieren: Das vorlegende Gericht muss natürlich darlegen, warum eine bestimmte Frage der Auslegung oder Gültigkeit von Unionsrecht für das Ergebnis des Ausgangsfalles überhaupt entscheidungserheblich ist. Dies kann das Herausarbeiten möglicher Auslegungsoptionen des in Rede stehenden Unionsrechts notwendig machen. Typischerweise erklärt das nationale Gericht dabei, dass Auslegung A zum Ergebnis X und Auslegung B zum Ergebnis Y führen wird. Und nicht selten äußern die Gerichte durchaus auch ihre Präferenz für Auslegung A oder B. Normalerweise betonen sie allerdings, Zweifel im Hinblick auf die korrekte Auslegung zu haben. Von Zweifeln ist beim BVerfG nichts zu erkennen.

Ein weiterer Gesichtspunkt: Der EuGH könnte schon unter Hinweis auf die der Ultra vires-Doktrin des BVerfG zugrunde liegende Logik die Vorlage verwerfen. Möglicherweise liegt schon deswegen keine echte Vorlagefrage im Sinne des Art. 267 AEUV vor, weil das nationale Gericht mit seiner Ultra vires-Doktrin einen

47 Vgl. hierzu EuG, Beschl. v. 10.12.2013, Rs. T-492/12 (von Storch u.a./EZB), Slg. 2013, II-00000, in dem das EuG eine Klagebefugnis zur Klage gegen das OMT-Programm der EZB von Individuen vor den europäischen Gerichten in diesem Zusammenhang verneint. Zu den Fragen der Zulässigkeit vgl. im Einzelnen auch M. Wendel, Kompetenzrechtliche Grenzgänge, ZaöRV 2014, i.E.

48 BVerfG, 2 BvR 2728/13, Beschl. v. 14.1.2014 - OMT, Rn. 101.

49 Der EuGH beantwortet keine Fragen, die ihm im Rahmen konstruierter Verfahren vorgelegt werden, mit deren Hilfe die Parteien den Gerichtshof zur Stellungnahme zu europarechtlichen Fragen veranlassen wollen, deren Beantwortung für die Entscheidung eines Rechtsstreits nicht objektiv erforderlich ist, EuGH, Rs. 244/80 (Foglia), Slg. 1981, 3045.

50 Vgl. zu diesem Punkt das Sondervotum Lübbe-Wolff, BVerfG, 2 BvR 2728/13, Beschl. v. 14.1.2014 - OMT, Sondervotum Lübbe-Wolff, Rn. 11. 
Letztentscheidungsanspruch in Bezug auf Unionsrecht geltend macht. Insoweit kommt es auf die Vorlageantwort des EuGH gar nicht mehr an. Sollte der EuGH auf die vom BVerfG vorgelegten Fragen antworten, ohne auf den Anspruch auf Letztentscheidung einzugehen, riskiert der EuGH, dass nationale Gerichte Letztentscheidungsansprüche über Europarecht, etwa im Rahmen einer Ultra viresKontrolle, anerkannt sehen bzw. sich zu solchen Ansprüchen ermutigt fühlen.

Aufgrund der Bedeutung und der Sichtbarkeit des Falles erscheint es freilich in hohem Maße unwahrscheinlich, dass der EuGH die erste Vorlage des BVerfG überhaupt schlicht wegen Unzulässigkeit der Vorlagefrage nach Deutschland zurückschickt. Unwahrscheinlich - aber nicht völlig ausgeschlossen. Im Verfahren vor dem EuGH haben jedenfalls etliche der äußerungsberechtigten Mitgliedstaaten Zulässigkeitszweifel erkennen lassen.

\section{Unter welchen Voraussetzungen würde das BVerfG das OMT-Programm als rechtmäßig erachten?}

Der Vorlagebeschluss fasst diese Voraussetzungen in Randnummer 100 zusammen. Im Vordergrund stehen dabei die Begrenzung des OMT-Programms in seinem Umfang, der Ausschluss von Schuldenschnitten sowie der Schutz der Bildung der Marktpreise für Anleihen. In den Worten der Senatsmehrheit: Das OMT-Programm darf nicht ,die Konditionalität der Hilfsprogramme von Europäischer Finanzstabilisierungsfaszilität und Europäischem Stabilitätsmechanismus“ unterlaufen; es muss ,einen die Wirtschaftspolitik der Union nur unterstützenden Charakter“ aufweisen, was ,[m] it Blick auf Art. 123 AEUV [voraussetzt], dass ein Schuldenschnitt ausgeschlossen werden muss, Staatsanleihen einzelner Mitgliedstaaten nicht in unbegrenzter Höhe angekauft werden und Eingriffe in die Preisbildung am Markt soweit wie möglich vermieden werden".

Vieles spricht dafür, dass das OMT-Programm mit solchen Fesseln versehen nicht mehr effektiv funktionieren würde und die EZB ernsthaft in ihrer Handlungsfähigkeit eingeschränkt wäre. ${ }^{51}$ Möglicherweise sind aber einzelne oder alle der im Beschluss genannten Kriterien und Gesichtspunkte einer flexiblen Auslegung zugänglich. Zur Beschränkung des Umfangs eines Anleiheaufkaufprogramms könnte beispielsweise die allgemeine Beschreibung einer sehr hohen Schranke in einer hypothetischen Größenordnung einerseits genug Glaubwürdigkeit und Handlungsfähigkeit bei der EZB belassen und zugleich formal dem Kriterium des BVerfG, Staatsanleihen dürften ,nicht in unbegrenzter Höhe“ gekauft werden, entsprechen. Hier ist daran zu erinnern, dass das BVerfG sich selbst sehr schwer getan hat, Grenzen für den Umfang der Rettungsschirme (EFSF, jetzt ESM) zu benennen und sich für diese Grenze letztlich auf einen Offenkundigkeitstest zurückzieht. ${ }^{52}$

51 M. Fratzscher, Ein Richterspruch mit Risiko, DIE ZEIT online v. 7.2.2014, abrufbar unter: http://www.zeit.de/ wirtschaft/2014-02/gastbeitrag-fratzscher-BVerfG-ezb.

52 BVerfGE 129, 124 - Griechenlandhilfe/EFSF. 
Der EuGH könnte ferner darauf verweisen, dass das OMT-Programm sich als im Tatsächlichen begrenzt darstellt, weil das Programm innerhalb eines bestimmten Mandates erfolgt und über die Anbindung an ein EFSF- oder ESM-Programm ohnehin weiteren Bindungen unterliegt. Gleichzeitig müsste der EuGH freilich betonen, dass die EZB aufgrund ihrer besonderen Funktion als unabhängige Zentralbank $^{53}$ diese Grenzen grundsätzlich selbst definiert und darüber hinaus ausschließlich der EuGH ausschließlich in offenkundigen Ausnahmefällen die Grenzen des Handelns der EZB mit Marktbezug ${ }^{54}$ bewerten kann. Mit anderen Worten: Wahrscheinlich wird der EuGH zu den in Randnummer 100 niedergelegten Bedingungen bestätigende Formeln suchen, ohne neue Hindernisse für den Spielraum der EZB zu schaffen. Damit würde die Funktionsfähigkeit der EZB gewahrt und zugleich dem BVerfG ein einigermaßen gesichtswahrender Ausweg eröffnet. Um auf die Metapher des eingangs erwähnten Films von 1957 zurückzukommen, das Hasenfußrennen: Vieles spricht dafür, dass es kein Rennen geben muss. Das BVerfG ist mit seinem Interpretationsdiktat und der Drohung, das OMT-Programm aufzuhalten, in gewissem Sinne alleine losgerast. Es droht der Absturz in den argumentativen Abgrund. Das wäre die offene Konfrontation des BVerfG mit EuGH und EZB, mit dem Risiko nachhaltiger Schäden an Recht und Institutionen. Um diesen Verlauf zu vermeiden braucht das BVerfG die Hilfe des EuGH, um aus dem Wagen noch rechtzeitig auszusteigen.

\section{Wird der EuGH innerhalb von wenigen Wochen entscheiden?}

Nein. Vorabentscheidungsverfahren können zwischen wenigen Wochen, wenn es um Leib, Leben und Gesundheit geht und - so derzeit der Durchschnitt - 17 Monaten dauern. ${ }^{55}$ Nach Art. 267 Abs. 4 AEUV entscheidet der EuGH in Fällen, die eine inhaftierte Person betreffen, innerhalb kürzester Zeit. Die Beschleunigung wird in der Regel vom vorlegenden Gericht ausgehen. Nach Art. 105 ff. der Verfahrensordnung des EuGH kann nämlich das vorlegende nationale Gericht ein beschleunigtes Verfahren beantragen. In der den ESM-Vertrag betreffenden Pringle-Vorlage beantragte der irische Supreme Court ein solches beschleunigtes Verfahren, was zu einer Entscheidung des EuGH in weniger als vier Monaten führte. ${ }^{56}$ Das BVerfG hat von dieser Beschleunigungsmöglichkeit keinen Gebrauch gemacht. Abgesehen von der durchaus bestehenden Möglichkeit des EuGH, dies anders zu handhaben,

53 Vgl. Art. 130 AEUV. Die Unabhängigkeit der EZB wird sogar in Art. 88 GG erwähnt und ist gerade von Deutschland bei der Konzeption der Währungsunion eingefordert worden, wie die Entstehungsgeschichte der Wirtschafts- und Währungsunion belegt. Näher dazu H. James, Making the European Monetary Union. The Role of the Committee of Central Bank Governors and the Origins of the European Central Bank, 2012, S. $270 \mathrm{ff}$.

54 Der EuGH hat bereits entschieden, dass die EZB nicht außerhalb des Unionsrechts steht, wenn es beispielsweise um die Bindung an das Betrugsbekämpfungsregime OLAF geht, EuGH, Rs. C-11/00 (Kommission/EZB), Slg. 2003, I-7147.

55 Zwischen 2008 und 2012 lag die durchschnittliche Verfahrensdauer von Vorabentscheidungen bei 16,4 Monaten, vgl. den Jahresbericht des Gerichtshofes der Europäischen Union, abrufbar unter: http://curia.europa.eu/ $\mathrm{jcms} / \mathrm{jcms} / \mathrm{Jo} 2$ _7000/.

56 EuGH, Rs. C-370/12 (Pringle), Slg. 2012, I-00000. 
wird der Fall daher mehr oder weniger wie ein gewöhnliches Vorabentscheidungsverfahren behandelt. Deswegen ist bis auf weiteres mit einem EuGH-Urteil irgendwann spät im Jahre 2014 oder, wahrscheinlicher, bis Mitte 2015 zu rechnen.

\section{Wird es zu einer weiteren mündlichen Verhandlungsschlacht kommen?}

Nein. Mündliche Verhandlungen vor dem EuGH unterscheiden sich in mehreren Punkten von mündlichen Verhandlungen vor dem BVerfG. Im Fall von ESM- und Fiskalvertrag war die mündliche Verhandlung vor dem BVerfG ein zweitägiger Medien- und Sachverständigenmarathon mit nach außen gerichtetem Schaukampfcharakter, an dem sowohl Mitglieder der Bundesregierung, der Präsident der Bundesbank, ein Mitglied des EZB-Rates, als auch über die Zukunft des Euro streitende Ökonomen beteiligt waren und über den Medien aus der ganzen Welt berichteten. Dabei hatte es für die Eilentscheidung bereits einen ähnlichen Medientrubel gegeben. Vor dem EuGH ist das Verfahren nüchterner und formeller. ${ }^{57}$ Insoweit ergeben sich selten neue Gesichtspunkte über die bereits eingereichten schriftlichen Stellungnahmen hinaus. Es treten zudem grundsätzlich nur die Rechtsbeistände der Parteien bzw. der Mitgliedstaaten auf. Minister oder gar Regierungschefs, sonstige Politiker oder Sachverständige sind gewöhnlich nicht beteiligt.

\section{Kann die EZB das OMT-Programm immer noch aktivieren?}

Ja, da das BVerfG weder versucht hat, selbst eine gegen das OMT-Programm gerichtete einstweilige Anordnung zu treffen, noch den EuGH gebeten hat, einstweilige Maßnahmen gegenüber der EZB anzuordnen. Rechtlich wird die EZB durch nichts daran gehindert, das OMT-Programm jederzeit einzuleiten, falls sie es für notwendig hält.

\section{Sind Anleiheaufkäufe im Rahmen eines Quantitative easing ebenfalls ein Problem?}

„Quantitative Easing“(QE, Quantitative Lockerung) ist ein weiteres Instrument von Zentralbanken, bei dem diese Anleihen auf dem Sekundärmarkt erwerben. Anders als das OMT-Programm wäre es nicht auf bestimmte, in Schwierigkeiten geratene Mitgliedstaaten beschränkt. Stattdessen würden die EZB und die nationalen Zentralbanken in erheblichem Umfang Anleihen aller Eurostaaten kaufen.

In den Verhandlungen vor dem BVerfG wurde diese Frage nicht im Einzelnen diskutiert. Quantitative Easing könnte aber die nächste Maßnahme sein, der sich die EZB zuwenden muss oder zuwenden will. ${ }^{58}$ Ausführungen von EZB-Präsident

57 Dies mag nicht zuletzt an den Erfordernissen der Simultanübersetzung liegen, die es angeraten sein lässt, möglichst vom Blatt vorzutragen.

58 Vgl. z.B. D. Oakley, Bond markets weigh risks of eurozone QE, FT v. 18.2.2014. 
Draghi im August 2014 auf einer Zusammenkunft von Zentralbankern in Jackson Hole, USA, wurden verbreitet als Ankündigung von QE gedeutet. ${ }^{59} \mathrm{Im}$ Vordergrund steht dabei die Frage wirksamer Maßnahmen gegen eine Deflationsentwicklung, aber es wird auch wieder die Frage gestellt werden, ob mit diesem Instrument nicht bestimmten Mitgliedstaaten eine Ausgabenpolitik über die eigenen Verhältnisse ermöglicht wird. ${ }^{60}$ Politische wie rechtliche Auseinandersetzungen um eine solche Maßnahme der EZB sind absehbar. Möglicherweise werden wieder diejenigen, die in der Vergangenheit regelmäßig das BVerfG eingeschaltet haben, auch QE angreifen.

Die in Randnummer 100 der OMT-Vorlage des BVerfG entwickelten Kriterien geben für die Prüfung eines von der EZB eingerichteten QE-Programms wenig her es würde sich eben auch rechtlich bei QE um einen neuen Fall handeln. Man darf dabei Folgendes nicht aus dem Blick verlieren: Der Erwerb von Staatsanleihen ist der EZB wie auch den nationalen Zentralbanken nicht verboten. Das Vertragsrecht geht sogar davon aus, dass die EZB dieses Instrument zur Verfügung hat. Art. 123 AEUV legt fest, dass „der unmittelbare Erwerb von Schuldtiteln“61 von Mitgliedstaaten verboten ist. Das Wort „unmittelbar“" würde keinen Sinn ergeben, wenn es nicht möglich wäre, Schuldtitel mittelbar, nämlich auf dem Sekundärmarkt, zu erwerben. Darüber hinaus definiert sogar das BVerfG Währungspolitik als auf die gesamte Eurozone bezogen und beanstandet beim OMT-Programm gerade, dass es nur für einzelne Euro-Mitgliedstaaten gilt (Selektivität). ${ }^{62} \mathrm{QE}$ würde für die gesamte Eurozone gelten und einzelne Mitgliedstaaten nicht bevorzugen (Nichtselektivität). Dies könnte darauf hinzuweisen, dass QE auch aus Sicht des BVerfG anders zu beurteilen ist als das OMT-Programm. Letztlich kommt es hier auf die konkrete Ausgestaltung eines Ankaufprogramms an. Dabei ist auch zu bedenken, dass zwischen einem OMT-Programm und einem QE-Programm konzeptionelle Unterschiede bestehen. Das OMT-Programm richtete sich gegen steigende Zinsausschläge bei Staatsanleihen einzelner Eurostaaten, um diesen Staaten eine Refinanzierung am Kapitalmarkt zu ermöglichen. Dieses Ziel konnte bereits mit einer bloßen Ankündigung erreicht werden, wobei das Offenlassen des Volumens (,,whatever it ta$\mathrm{kes}^{6}$ ) eine Rolle spielte. Ein QE-Programm reagiert auf die allgemeine wirtschaftliche Entwicklung (Deflation u.ä.) und soll auf diese einwirken. Dementsprechend würde falls gesamtwirtschaftlich erforderlich QE einfach mit einem bestimmten Volumen durchgeführt. QE ist insoweit ein gegenüber einem Programm wie dem OMT-Programm grundverschiedenes Zentralbankinstrument.

Der Blick in die Vorgeschichte des Art. 123 AEUV zeigt freilich, dass schon bei den Vorarbeiten für die Vertragsgrundlagen der Währungsunion durch das Com-

59 S. etwa S. Nixon, ECB's Draghi Takes a Gamble on QE-lite, Wall Street Journal v. 7.9.2014.

60 Kritisch zum Beispiel P. A. Fischer, „Quantitative Easing“ der EZB. Geldpolitik in den Fängen der Schuldenwirtschaft (Kommentar), NZZ v. 12.4.2014, S. 25.

61 Hervorhebung hinzugefügt.

62 BVerfG, 2 BvR 2728/13, Beschl. v. 14.1.2014 - OMT, Rn. 73. 
mittee of Governors ${ }^{63}$ Uneinigkeit darüber bestand, ob das im anglo-amerikanischen Raum etablierte Zentralbankinstrument der „open market operations“ der neuen europäischen Zentralbank überhaupt zur Verfügung stehen sollte oder nicht. ${ }^{64}$ Die Beschränkung auf Sekundärmarktoperationen im Kontext der Währungspolitik erweist sich damit als Kompromissformel. Es wird wieder auf das Verständnis der Reichweite von Währungspolitik ankommen.

\section{Was wird als nächstes passieren - wird das BVerfG jede Entscheidung des EuGH hinnehmen?}

Wahrscheinlich nicht. Es bestehen im Kern drei Szenarien:

- Nach einem ersten Szenario würde der EuGH nachgeben und die Auslegung des Unionsrechts durch das BVerfG im Sinne von Randnummer 100 der Vorlage vollumfänglich übernehmen. Dieses Szenario ist nicht sehr wahrscheinlich, schon weil die Argumentation des BVerfG auf rechtlicher Ebene nicht überzeugt. Die Unabhängigkeit der EZB nach Art. 130 AEUV sowie die vom Wortlaut des Art. 123 AEUV ausgesparten Aktivitäten der EZB auf dem Sekundärmarkt sind dabei die gewichtigsten Gegenargumente. ${ }^{65}$

- Das entgegengesetzte Szenario wäre die Verwerfung der Vorlage durch den EuGH bereits als unzulässig, ohne auf die Vorlagefrage auch nur näher einzugehen. ${ }^{66}$ Dies erscheint nicht völlig ausgeschlossen, aber doch eher unwahrscheinlich. Eine solche Brüskierung bei der allerersten Vorlage eines nationalen Höchstgerichts wäre nicht nur äußerst unfreundlich, es würde auch die Kooperation der Gerichte nachhaltig beschädigen.

- Das wahrscheinlichste Szenario ist, dass der EuGH einen Kompromiss zwischen Wahrung der Unabhängigkeit und der Handlungsspielräume der EZB einerseits und der Eröffnung eines gesichtswahrenden Ausweges für das BVerfG andererseits suchen wird.

Im Falle des zweiten oder dritten Szenarios haben die sechs Richter der Senatsmehrheit zu entscheiden, wie mit der Divergenz zwischen der im Vorlagebeschluss geäußerten Einschätzung des BVerfG und der Vorlageantwort des EuGH umzugehen ist. Die beiden abweichenden Richter haben das BVerfG bereits verlassen. Die zwölfjährige Amtszeit der Richterin Lübbe-Wolff endete im Juni,, die Amtszeit des Richters Gerhardt im Juli 2014. Die diesen beiden nachfolgenden Richter, Doris König und Ulrich Maidowski, werden bei der Fortführung des Falles in Deutschland nicht mitwirken. Es wird also darum gehen, welche Mehrheitsposition sich unter den verbleibenden sechs Richtern entwickelt.

63 Ausschuss der Präsidenten der Zentralbanken der Mitgliedstaaten, Gouverneursausschuss.

64 H. James, Making the European Monetary Union. The Role of the Committee of Central Bank Governors and the Origins of the European Central Bank, 2012, S. 287.

65 S. BVerfG, 2 BvR 2728/13, Beschl. v. 14.1.2014-OMT, Sondervoten, für die Argumente gegen die Begründung der Mehrheit.

66 S.o. FAQ Nr. 4. 
Aus der Sicht des Unionsrechts ist dabei völlig klar, dass Entscheidungen des EuGH nach Art. 267 AEUV nicht lediglich freundliche Empfehlungen sind, welchen das nationale Gericht folgen kann oder auch nicht. Die Rechtsprechung des EuGH ist in diesem Punkt eindeutig. Danach ,entscheidet ein Urteil, in dem der Gerichtshof im Wege der Vorabentscheidung über die Auslegung oder die Gültigkeit einer Handlung eines Gemeinschaftsorgans befindet, mit Rechtskraft über eine oder mehrere Fragen des Gemeinschaftsrechts und bindet das nationale Gericht bei seiner Entscheidung des Ausgangsrechtsstreits. “67

Eine Vorlageentscheidung des EuGH nicht oder nicht vollständig zu befolgen wäre demnach ein Verstoß gegen das Unionsrecht. Verletzungen des Unionsrechts werden Deutschland als Mitgliedstaat auch dann zugerechnet, wenn es um Handlungen oder Unterlassungen unabhängiger Gerichte geht. ${ }^{68}$ Es würde daher mit hoher Wahrscheinlichkeit gegen Deutschland ein Vertragsverletzungsverfahren eingeleitet, jedenfalls wenn das BVerfG die Entscheidung des EuGH offen missachten sollte. Es gilt der allgemeine Grundsatz pacta sunt servanda, vertragliche Verpflichtungen sind einzuhalten. Der EU-Vertrag normiert in Art. 4 EUV, dass die Mitgliedstaaten alle geeigneten Maßnahmen allgemeiner oder besonderer Art zur Erfüllung der Verpflichtungen, die sich aus den Verträgen oder den Handlungen der Organe der Union ergeben, ergreifen müssen. Die Mitgliedstaaten haben auch jegliche Maßnahmen zu unterlassen, welche die Ziele der Union gefährden könnten. Im vorliegenden Kontext betont Art. 131 AEUV, dass die Mitgliedstaaten sicherzustellen haben, dass die nationalen Rechtsordnungen nicht mit den Handlungen der EZB unvereinbar sind.

Das BVerfG insistiert in seiner Ultra vires-Argumentation freilich auf einer anderen, eigenen Perspektive und besteht darauf, dass die Einordnung eines Unionsaktes - hier ein Akt der EZB - als Kompetenzüberschreitung die Reichweite der Kompetenzübertragung von Deutschland an die EU betrifft, die sich nach deutschen Verfassungsrecht bemesse. Danach würde eine Bestätigung der Vereinbarkeit des - aus deutscher Sicht kompetenzwidrigen - OMT-Programms mit Unionsrecht seitens des EuGH durch das BVerfG wahrscheinlich ebenfalls als Ultra vires-Akt, dann des EuGH, angesehen.

Daneben würde das BVerfG möglicherweise argumentieren, dass unabhängig von einer Kompetenzüberschreitung die vertraglichen Verpflichtungen Deutschlands im Sinne des Art. 4 EUV jedenfalls ihre verfassungsrechtlichen Grenzen in der

67 EuGH, Rs. 69/85 (Wünsche), Slg. 1986, 947 Rn. 13.

68 Das Europarecht nimmt insoweit einen völkerrechtlichen Blickwinkel gegenüber den Mitgliedstaaten ein. Das Völkerrecht gestattet im Grundsatz keine Völkerrechtsverletzung aus Gründen des innerstaatlichen Rechts, s. bereits PCIJ, Treatment of Polish Nationals and Other Persons of Polish Origin or Speech in the Danzig Territory, PCIJ Serie A/B Nr. 44, 22. S. für einen Präzedenzfall einer durch deutsche Gerichte veranlassten Einleitung eines Vertragsverletzungsverfahren gegen Deutschland den Fall Hendrix („Pingo-Hähnchen“), Vorverfahren gem. Art. 169 EG-Vertrag (heute Art. 258 AEUV), A/90/0406, begründete Stellungnahme der Kommission SG (90)/D/25672 v. 3.8.1990, Teil 5 (hinsichtlich des BGH). S. zu einem vergleichbaren Fall eines Vertragsverletzungsverfahrens gegen Schweden E. Lenski/F. C. Mayer, Vertragsverletzung wegen Nichtvorlage durch oberste Gerichte, EuZW 2005, S. 225. 
Verfassungsidentität des Grundgesetzes finden, ${ }^{69}$ aus dieser Perspektive ebenfalls eine Frage des deutschen Verfassungsrechts, die folglich in die Zuständigkeit des BVerfG fällt.

Fraglos würde ein offener Konflikt zwischen den Gerichten am Ende das Ansehen beider Gerichte und überdies die Rechtsgemeinschaft in der EU nachhaltig beschädigen. Deswegen ist ein solcher Verlauf eines weiteren „Hasenfußrennens“, nun zwischen BVerfG und dem EuGH, in hohem Maße unwahrscheinlich.

\section{Was wären die Konsequenzen, wenn das BVerfG einen EU-Akt als ultra vires bezeichnen würde?}

Im Maastricht-Urteil von 1993 formuliert das BVerfG die Konsequenzen einer Ultra vires-Feststellung wie folgt:

„,Würden etwa europäische Einrichtungen oder Organe den Unions-Vertrag in einer Weise handhaben oder fortbilden, die von dem Vertrag, wie er dem deutschen Zustimmungsgesetz zugrunde liegt, nicht mehr gedeckt wäre, so wären die daraus hervorgehenden Rechtsakte im deutschen Hoheitsbereich nicht verbindlich. Die deutschen Staatsorgane wären aus verfassungsrechtlichen Gründen gehindert, diese Rechtsakte in Deutschland anzuwenden. Dementsprechend prüft das BVerfG, ob Rechtsakte der europäischen Einrichtungen und Organe sich in den Grenzen der ihnen eingeräumten Hoheitsrechte halten oder aus ihnen ausbrechen (vgl. BVerfGE 58, 1 [30f.]; 75, 223 [235, 242]). “70

Beschwerdeführer und Antragsteller argumentieren, dass die Bundesregierung und der Deutsche Bundestag im Falle einer entsprechenden Ultra vires-Feststellung durch das BVerfG verpflichtet seien, auf die Aufhebung des in Frage stehenden Ultra vires-Aktes d.h. - hier die OMT-Entscheidung - hinzuwirken bzw. dessen Umsetzung zu verhindern. Es ist reichlich unklar, wie ein dies bewirkendes Urteil des BVerfG konkret formuliert sein könnte. Eine Anweisung des BVerfG an Bundesregierung und Bundestag, auf die Aufhebung des OMT-Programms hinzuwirken

69 S. hierzu unten, FAQ Nr. 13.

70 BVerfGE 89, 155, 188 - Maastricht. S. auch die einschlägige Passage der Honeywell-Entscheidung: „Anders als ein bundesstaatlicher Geltungsvorrang, wie ihn Art. 31 GG für die deutsche Rechtsordnung vorsieht, kann der Anwendungsvorrang des Unionsrechts nicht umfassend sein (vgl. BVerfGE 73, 339 [375]; 123, 267 [398]). Das Unionsrecht bleibt als autonomes Recht von der vertraglichen Übertragung und Ermächtigung abhängig. Die Unionsorgane bleiben für die Erweiterung ihrer Befugnisse auf Vertragsänderungen angewiesen, die von den Mitgliedstaaten im Rahmen der für sie jeweils geltenden verfassungsrechtlichen Bestimmungen vorgenommen und verantwortet werden (vgl. BVerfGE 75, 223 [242]; 89, 155 [187 f., 192, 199]; 123, 267 [349]; vgl. auch BVerfGE 58, 1 [37]; 68, 1 [102]; 77, 170 [231]; 104, 151 [195]; 118, 244 [260]). Es gilt das Prinzip der begrenzten Einzelermächtigung (Art. 5 Abs. 1 Satz 1 und Abs. 2 Satz 1 EUV). Das BVerfG ist deshalb berechtigt und verpflichtet, Handlungen der europäischen Organe und Einrichtungen darauf zu überprüfen, ob sie aufgrund ersichtlicher Kompetenzüberschreitungen oder aufgrund von Kompetenzausübungen im nicht übertragbaren Bereich der Verfassungsidentität (Art. 79 Abs. 3 i.V.m. Art. 1 und Art. 20 GG) erfolgen (vgl. BVerfGE 75, 223 [235, 242]; 89, 155 [188]; 113, 273 [296]; 123, 267 [353f.]), und gegebenenfalls die Unanwendbarkeit kompetenzüberschreitender Handlungen für die deutsche Rechtsordnung festzustellen.“, BVerfGE 126, 286, 302 Honeywell. 
ist schon deswegen ein merkwürdiger Gedanke, weil nationale Regierungen oder Parlamente der EZB keinerlei Weisungen erteilen können. Jedenfalls ist klar, dass das BVerfG die Bundesregierung nicht anweisen kann, den Austritt aus dem Euro einzuleiten, weil ein solcher Austritt in den Verträgen nicht vorgesehen ist.

Das Sondervotum Lübbe-Wolff lässt erkennen, dass dieser Punkt im Senat kontrovers diskutiert worden ist und äußert sich mehr als skeptisch:

„,Vermutlich ist das angesprochene Sich-Befassen von Bundestag und Bundesregierung nicht als einzige einklagbare Reaktion auf ein etwaiges qualifiziertes ultra-vires-Handeln oder eine Verletzung der deutschen Verfassungsidentität durch die Europäische Zentralbank gedacht. Für die Lesart, dass gegebenenfalls weitere Reaktionen einforderbar sein sollen, sprechen zumindest die Randnummern 44 und 50. Was aber genau verlangt werden kann (auch das Ausscheiden aus der Währungsgemeinschaft?) und in welcher Weise das Beanspruchbare zu geschehen hat (alternativ? kumulativ? sukzessiv? in welcher Abfolge?), wird nicht deutlich. Angesichts des Mangels an Rechtsquellen, aus denen sich eine Antwort auf diese Fragen schöpfen ließe, ist das nur zu verständlich. Nur sollte man sich auf große Wüstenwanderungen, die zu keiner Quelle führen, gar nicht erst schicken lassen. "71

Möglicherweise läuft all dies letztlich lediglich darauf hinaus, dass das BVerfG den Bundestag verpflichtet, das OMT-Programm zum Gegenstand einer Plenardebatte zu machen ${ }^{72}$ - eine merkwürdige Konstruktion im Hinblick auf Gewalten- und Rollenteilung zwischen BVerfG und Parlament.

Vielleicht müsste das hier streitige Konstrukt einmal in einem anderen aktuelleren Kontext getestet werden, bei dem es nicht bloß um vermutete und hypothetische Verläufe geht wie bei dem ja lediglich angekündigten OMT-Programm. Um bereits erlittene reale Souveränitäts-, Grundrechts- und Rechtsschutzeinbußen geht es bei Aktivitäten der NSA in Deutschland, ${ }^{73}$ und es stellt sich die Frage, ob entsprechend der Linie des BVerfG im OMT-Beschluss womöglich Individuen das BVerfG auch anrufen können um zu erreichen, dass das BVerfG die Regierung oder das Parlament anweist, konkrete Maßnahmen in der NSA-Frage zu ergreifen.

Nur weil etwas nicht nützt, heißt das nicht, dass es nicht doch schadet: Hier darf nicht unterschätzt werden, worauf der abweichende Richter Gerhardt in diesem Kontext aufmerksam macht: „Über die konsequenterweise eingeräumte Möglichkeit, vorbeugenden Rechtsschutz einzufordern, können einzelne Wahlberechtigte das BVerfG zu Zeitpunkten, in denen der politische Prozess noch andauert, ins Spiel bringen"."74

71 BVerfG, 2 BvR 2728/13, Beschl. v. 14.1.2014 - OMT, Sondervotum Lübbe-Wolff, Rn. 23, Hervorhebungen im Original.

72 BVerfG, 2 BvR 2728/13 Beschl. v. 14.1.2014 - OMT, Rn. 53.

73 Vgl. dazu F. C. Mayer, Mit Europarecht gegen Abhöraktionen?, http://www.verfassungsblog.de/de/mit-europarecht-gegen-die-amerikanischen-und-britischen-abhoeraktionen-teil-1-nsa.

74 BVerfG, 2 BvR 2728/13, Beschl. v. 14.1.2014 - OMT, Sondervotum Gerhardt, Rn. 7. 
Es besteht eine weitere mögliche Konsequenz aus der Feststellung durch das BVerfG, dass das OMT-Programm einen Ultra vires-Akt darstellt. Diese wird in den Sondervoten gar nicht und in der Entscheidung selbst ${ }^{75}$ eher knapp erwähnt, fast so als ob die Richter davor zurückscheuen, diese Büchse der Pandora zu öffnen. Sie wird freilich von den Beschwerdeführern und der Antragstellerin im OMTVerfahren deutlich adressiert. ${ }^{76}$ Wenn ein Ultra vires-Akt einer EU-Institution ,,im deutschen Hoheitsbereich nicht verbindlich“ wäre und die „deutschen Staatsorgane [...] aus verfassungsrechtlichen Gründen gehindert [wären], diese Rechtsakte in Deutschland anzuwenden"77, dann käme es entscheidend auf die eine deutsche Institution an, die bei den OMT eine Rolle spielt. Dies ist die Bundesbank, weil der Ankauf von Staatsanleihen auf dem Sekundärmarkt unter dem OMT-Programm technisch durch die nationalen Zentralbanken als Teil des Eurosystems abgewickelt wird. 78

Konkret: Die Bundesbank könnte durch das BVerfG verpflichtet werden ${ }^{79}$ - oder auch ohne eine ausdrückliche Anweisung sich verpflichtet fühlen -, an der Umsetzung des OMT-Programms nicht mitzuwirken, soweit dieses Programm durch das BVerfG als Ultra vires-Akt angesehen wurde.

Eine eindeutige rechtliche Aussage dazu, welches Recht - das europäische oder das deutsche - für die Bundesbank als ausführende Einrichtung, die der EZB nachgeordnet ist, im Konfliktfall zu befolgen ist, ist nicht ohne weiteres zu treffen.

Die Bundesbank ist eine unabhängige Zentralbank und ist damit Anweisungen des BVerfG nicht ohne weiteres ausgesetzt. Art. 130 AEUV bestimmt, dass nationale Zentralbanken bei der Wahrnehmung der ihnen durch die Verträge und die Satzung des ESZB und der EZB übertragenen Befugnisse, Aufgaben und Pflichten - hier konkret: Umsetzung des OMT-Programms - von keiner Regierung eines Mitgliedstaats oder einer anderen Stelle Weisungen entgegennehmen darf. Anerkanntermaßen geht EU-Recht dem nationalen Recht vor (Anwendungsvorrang), ${ }^{80}$ selbst dem Verfassungsrecht. ${ }^{81}$ Art. 131 AEUV bestimmt, dass die Mitgliedstaaten sicherstellen müssen, dass ihre innerstaatlichen Rechtsvorschriften in Einklang mit den Verträgen stehen. All dies könnte begründen, dass die Bundesbank unabhängig von einem Ausspruch des BVerfG an der Umsetzung des OMT-Programms mitwirkt. Sollte die Bundesbank sich aber weigern, am OMT-Programm teilzunehmen, dann würde dies zweifellos aus Sicht der europäischen Rechtsordnung einen Verstoß gegen das Europarecht bedeuten. Gemäß Art. 35 Abs. 6 ESZB/EZB-Statut könnte die EZB die Bundesbank dann vor den EuGH bringen.

77 BVerfGE 89, 155, 188 - Maastricht.

78 Näher dazu EZB, Stellungnahme v. 16.1.2013, S. 37, abrufbar unter http://www.handelsblatt.com/downloads/ 8135244/3/EZB\%20Gutachten.

79 BVerfG, 2 BvR 2728/13, Beschl. v. 14.1.2014-OMT, Rn. 45.

80 EuGH, Rs. 6/64 (Costa/ENEL), Slg. 1964, 1141.

81 EuGH, Rs. 11/70 (Internationale Handelsgesellschaft), Slg. 1970, 1125, 1134. 
Unabhängig von den rechtlichen Verpflichtungen würde man, falls die Bundesbank sich weigerte, die OMT-Ankäufe auszuführen, für die konkrete Abwicklung der Sekundärmarktankäufe wohl andere Lösungen versuchen. So könnten eine oder mehre andere Zentralbanken die Rolle übernehmen, die der deutschen Zentralbank zugedacht war und deren Teil an Sekundärmarktankäufen übernehmen. Deutschlands Haftung im Eurosystem bliebe davon freilich unberührt. Man muss bei einem solchen Szenario freilich Aspekte bedenken, die über eine lediglich rechtliche Betrachtung hinausführen. Bislang hatte das OMT-Programm auch ohne umgesetzt zu sein schon wegen der Psychologie der Märkte den gewünschten Effekt. Dies belegt, dass hier Grenzen formaler rechtlicher Argumente bestehen. Die psychologischen Effekte einer Weigerung der Zentralbank des größten Mitgliedstaates des Europäischen Systems der Zentralbanken, der EZB zu folgen, sind nicht absehbar und völlig unabhängig von den Rechtswegen bei einem Konflikt zwischen EZB und nationalen Zentralbanken. Offene Konflikte sogar zwischen den Zentralbanken würden letztlich die Glaubwürdigkeit und die Funktionsfähigkeit aller beteiligten Institutionen derart beschädigen, dass ein solcher Verlauf unwahrscheinlich erscheint.

\section{Was hat das OMT-Programm mit der deutschen Verfassungsidentität zu tun?}

Gegen Ende erwähnt die Vorlageentscheidung des BVerfG die „Verfassungsidentität des Grundgesetzes“، ${ }^{82}$ Dieses Konzept und seine konkrete Bedeutung für das OMT-Programm sind nicht ohne weiteres nachvollziehbar. Dabei spricht einiges dafür, dass es dieses Argument ist, dem sich die Senatsmehrheit zuwenden wird, sollte der EuGH es ablehnen, das OMT-Programm der EZB zum Ultra vires-Akt zu erklären.

Der Hintergrund ist dabei der folgende: Im Lissabon-Urteil 2009 hat das BVerfG einen neuen Ansatz formuliert, mit dem es Unionsrecht stoppen will, und zwar zusätzlich zur Ultra vires-Kontrolle, die auf die Maastricht-Entscheidung von 1993 zurückgeht. Diese „Identitätskontrolle“ wird im vierten Leitsatz des Lissabon-Urteils wie folgt zusammengefasst:

„,Darüber hinaus prüft das BVerfG, ob der unantastbare Kerngehalt der Verfassungsidentität des Grundgesetzes nach Art. 23 Abs. 1 Satz 3 in Verbindung mit Art. 79 Abs. 3 GG gewahrt ist (vgl. BVerfGE 113, $273<296>$ ). Die Ausübung dieser verfassungsrechtlich radizierten Prüfungskompetenz folgt dem Grundsatz der Europarechtsfreundlichkeit des Grundgesetzes, und sie widerspricht deshalb auch nicht dem Grundsatz der loyalen Zusammenarbeit (Art. 4 Abs. 3 EUV-Lissabon); anders können die von Art. 4 Abs. 2 Satz 1 EUV-Lissabon anerkannten grundlegenden politischen und verfassungsmäßigen Strukturen souveräner Mitgliedstaaten bei fortschreitender Integration nicht gewahrt werden. Insoweit ge- 
hen die verfassungs- und die unionsrechtliche Gewährleistung der nationalen Verfassungsidentität im europäischen Rechtsraum Hand in Hand. "83

Und:

„Die Identitätskontrolle ermöglicht die Prüfung, ob infolge des Handelns europäischer Organe die in Art. 79 Abs. 3 GG für unantastbar erklärten Grundsätze der Art. 1 und Art. 20 GG verletzt werden. Damit wird sichergestellt, dass der Anwendungsvorrang des Unionsrechts nur kraft und im Rahmen der fortbestehenden verfassungsrechtlichen Ermächtigung gilt. "84

Nach Art. 4 Abs. 2 EUV achtet die Europäische Union die nationale Identität der Mitgliedstaaten, inklusive der, ,grundlegenden politischen und verfassungsmäßigen Strukturen". Hier wird deutlich, dass nationale Identität auch Verfassungsidentität beinhaltet. Dies mag tatsächlich als Ansatzpunkt auf europäischer Ebene dazu dienen, den unbedingten Vorranganspruch des Unionsrechts mit der Schranke der jeweiligen nationalen Verfassungsidentität zu versehen.

Es verwundert nicht, dass es ein akademischer Beitrag aus Irland war, der aus dem Art. 4 Abs. 2 EUV inhärenten Schutz der grundlegenden nationalen Verfassungspräferenzen die Idee entwickelt hat, dass es die Rolle der nationalen letztinstanzlichen Gerichte sei, zu entscheiden, was genau zu diesen grundlegenden Entscheidungen gehört, die vom Unionsrecht anerkannt und geschützt sind. ${ }^{85}$ Es war nämlich das irische Protokoll zum Maastricht Vertrag von 1992, ${ }^{86}$ das zum ersten Mal die Idee kodifizierte, Verfassungsgehalte, die von einer besonderen Bedeutung für die betreffende Verfassung sind - hier das ausdrückliche Abtreibungsverbot der irischen Verfassung -, gleichsam europarechtsfest zu machen. Das Protokoll kann als eine Art Blaupause angesehen werden: Die Identitätsklausel des aktuellen EU-Vertrags nimmt den Ansatz aus dem irischen Protokoll auf und verallgemeinert ihn. Art. 4 Abs. 2 EUV lässt sich als eine Rücknahme des bedingungslosen Vorranganspruchs und damit auch als Grenze der Einheitlichkeit des Unionsrechts deuten, die im Hinblick auf bestimmte verfassungsrechtlich besonders hervorgehobene mit-

83 BVerfGE 123, 267 - Lissabon.

84 BVerfGE 123, 267, 253 ff. - Lissabon, Rn. 240.

85 D. R. Phelan, Revolt or Revolution, 1997, S. 416 f.: Um einen Aufstand oder eine (rechtliche) Revolution zu vermeiden und die Legitimität der nationalen Rechtsordnungen aufrechtzuerhalten, schlägt Phelan eine Ergänzung zu den Verträgen vor, welche den Vorrang von grundlegenden Prinzipien der mitgliedsstaatlichen Verfassungen gegenüber Europarecht sichert, und zwar bezüglich Leben, Freiheit, Religion und Familie, soweit es im Ausgangspunkt um die Person und Moral geht (und nicht um Markt und Güterverteilung). Die Rechte, in denen diese Prinzipien Ausdruck finden, würden innerhalb ihres Anwendungsbereichs als vorrangig zum Europarecht gelten. Der genaue Umfang dieses Vorbehalts würde von den jeweiligen nationalen Gerichten oder anderen letztinstanzlichen Institutionen entwickelt werden, S. 416, $417 \mathrm{f}$. Kritisch dazu M. Maduro, The Heteronyms of European Law, 5 ELJ 1999, S. 160, und N. MacCormick, Risking Constitutional Collision in Europe?, 18 Oxford Journal of Legal Studies 1998, S. 517; vgl. in diesem Kontext auch D. R. Phelan, The Right to Life of the Unborn v the Promotion of Trade in Services, 55 Modern Law Review 1992, S. 670.

86 17. Protokoll zum Maastricht-Vertrag. 
gliedsstaatliche Belange, die Teil der nationalen Verfassungsidentität sind, hinzunehmen ist. ${ }^{87}$

In den Mitgliedstaaten finden sich bereits vor dem Vertrag von Lissabon Ansätze, die die Idee einer nationalen Verfassungsidentität thematisieren und dabei nicht in Abwehrhaltung verharren, sondern die Idee nutzen, um eine Brücke zwischen dem europäischen und dem nationalen Verfassungsrecht zu bauen. So hat der französische Conseil Constitutionnel 2004 implizit und 2006 explizit erkennen lassen, ${ }^{88}$ dass er die nationale Verfassungsidentität bei der konstruktiven Suche nach einer Grenze für den Vorrang des Europarechts heranzieht. ${ }^{89}$

Ein ähnlicher, konstruktiver Ansatz findet sich auch in einer Entscheidung des spanischen Verfassungsgerichts von 2004. ${ }^{90}$ Sogar der EuGH hat 2010 begonnen, das Konzept der nationalen Verfassungsidentität aufzunehmen und es als Grenze der Reichweite des Unionsrechts zu thematisieren. ${ }^{91}$

Gerade für den vorliegenden Fall ist nicht unerheblich, dass ein grundlegender struktureller Unterschied zwischen Ultra vires-Akt und identitätsverletzendem Akt besteht. Der prinzipielle Schutz nationaler Verfassungsidentität beinhaltet nicht zwangsläufig einen Fehlervorwurf an die europäische Ebene und weist über das bipolare Verhältnis zwischen deutscher bzw. mitgliedstaatlicher und europäischer Rechtsordnung nicht hinaus. Anders die Konzeption des ausbrechenden Rechtsaktes bei der Frage nach der Einhaltung sachlicher Kompetenzschranken auf europäischer Ebene. Hier würde durch die Feststellung eines ausbrechenden Rechtsaktes das BVerfG stets zum Ausdruck bringen, dass es eine europäische Fehlentwicklung korrigiert und damit einen entsprechenden, mindestens impliziten, konfrontativen Fehlervorwurf an die Union und insbesondere den EuGH richten. Der Vorwurf eines kompetenzwidrigen Rechtsaktes betrifft zudem auch Geltung bzw. Anwendbarkeit des Europarechts in allen anderen Mitgliedstaaten. Hier geht es nicht mehr um eine bipolare Blickrichtung, weil eine Aussage über die Kompetenzmäßigkeit europäischen Handelns auch in alle anderen Mitgliedstaaten abstrahlt: Ein europäischer Rechtsakt kann nicht zugleich in Deutschland kompetenzwidrig und in anderen Mitgliedstaaten kompetenzgemäß sein - insoweit beansprucht die europäische Kompetenzordnung Einheitlichkeit, soweit sie nicht selbst Ausnahmen vorsieht. Bei Zweifelsfragen bedarf es dann auch einer einheitlichen Auslegung, und die kann nur das europäische Gericht sichern (Art. 19 EUV, Art. 344 AEUV). Hier hilft auch das Argument nicht weiter, die europäische Kompetenzordnung richte sich nach der

87 Vgl. zu dieser Idee vorausgehend zum Lissabon-Urteil F. C. Mayer, Kompetenzüberschreitung (Fn. 38), S. 341; ders., Verfassungsgerichtsbarkeit (Fn. 38), S. 424 f.; vgl. auch v. Bogdandy/Schill, Overcoming absolute primacy: Respect for national identity under the Lisbon Treaty, Common Market Law Review 2011, S. 1417; $M$. Wendel, Permeabilität im europäischen Verfassungsrecht, 2011, S. 572 ff.; M. Claes, Negotiating Constitutional Identity or Whose Identity is it Anyway?, in: dies. u.a. (Hrsg.), Constitutional Conversations in Europe, 2012, S. 205; C. Franzius, Art. 4 EUV, in: Nowak u.a. (Hrsg.), EUV/AEUV, iE., Rn. 31, 42.

88 Conseil Constitutionnel v. 19.11.2004, Traité établissant une Constitution pour l'Europe; Conseil Constitutionnel v. 27.6.2006, Loi relative au droit d'auteur et aux droits voisins dans la société de l'information.

89 S. hierzu M. Walter, Integrationsgrenze Verfassungsidentität, ZaöRV 2012, S. 177 (184 ff., 199).

90 Tribunal Constitucional, Urt. v. 13.12.2004, DTC 1/2004.

91 EuGH, Rs. 208/09 (Ilonka Sayn-Wittgenstein/Landeshauptmann von Wien), Slg. 2010, I-13693. 
Reichweite der jeweils übertragenen Kompetenzen. Dies kann nicht richtig sein, wenn es dann jeder Mitgliedstaat in der Hand hätte, die Reichweite seiner Verpflichtungen nach Belieben einseitig selbst zu definieren und zu variieren. Dies wäre keine Rechtsgemeinschaft mehr.

Auch die Entscheidung, was als Teil der nationalen Verfassungsidentität im Sinne des Art. 4 Abs 2 EUV anzusehen ist und deswegen Schranken des Unionsrechts ausmacht, kann nicht unilateral getroffen werden. Hier ist ein Zusammenwirken der nationalen und der europäischen Gerichte notwendig, weil es sowohl um die Auslegung des Unionsrechts (Art. 4 Abs. 2 EUV) als auch des nationalen Verfassungsrechts geht. ${ }^{92}$

Die den Vorlagebeschluss tragenden sechs Richter des Zweiten Senats scheinen in diese Richtung zu denken, wenn sie den EuGH in der OMT-Vorlageentscheidung im Kontext der Verfassungsidentität erwähnen. Genau besehen ist hier aber keine Rede von Kooperation. Die Richter erläutern, dass das BVerfG die Auslegung des relevanten Europarechts durch den EuGH in dessen Vorabentscheidung zugrunde legen wird, dass jedoch die „Feststellung des unantastbaren Kernbestandes der Verfassungsidentität und die Prüfung, ob die Maßnahme (in der vom Gerichtshof festgestellten Auslegung) in diesen Kernbestand eingreift" dem BVerfG obliege. ${ }^{93}$ Die sechs Richter geben sich dann große Mühe zu erklären, warum ,sich die Identitätskontrolle im Rahmen des Art. 79 Abs. 3 GG durch das BVerfG wesentlich von der Überprüfung des Art. 4 Abs. 2 Satz 1 EUV durch den Gerichtshof [unterscheidet] ${ }^{694}$.

Dieses Verständnis von Identitätskontrolle ist alles andere als hilfreich und auch nicht überzeugend. Das BVerfG hätte hier die gemeinsame Verantwortung beider Gerichtsebenen hervorheben sollen, anstatt bestehende Unterschiede $\mathrm{zu}$ betonen. Eine Pflicht zur - notfalls erneuten - Vorlage an den EuGH besteht, wenn ein mitgliedstaatliches Gericht unter Berufung auf die nationale Verfassungsidentität Unionsrecht unangewendet lassen will. Es geht dann nämlich um die Auslegung des Art. 4 Abs. 2 EUV. Zugleich kann der EuGH nicht letztverbindlich Gehalte mitgliedstaatlicher Verfassungsidentität definieren. Das Vorlageverfahren dient hier einem dialogischen Sichverständigen auf eine beiderseits akzeptable Grenzlinie.

Aber was hat das OMT-Programm mit all dem zu tun?

Wenn das BVerfG mit seiner Ultra vires-Argumentation beim EuGH nicht durchdringt, so könnte die Senatsmehrheit auf die Identitätskontrolle zurückgreifen, um das OMT-Programm in Deutschland für unanwendbar zu erklären. Das Argument wäre dann, dass wegen eines Übergriffs in die Verfassungsidentität des Grundge-

92 Detaillierter hierzu F. C. Mayer/M. Wendel, Die verfassungsrechtlichen Grundlagen des Europarechts, in: Hatje/ Müller-Graff(Hrsg.), Enzyklopädie des Europarechts, Band 1, 2014, S. 163; dies., Multilevel Constitutionalism and Constitutional Pluralism - querelle allemande or querelle d'Allemand?, in: Avbelj/Komárek (Hrsg.), Constitutional Pluralism in the European Union and Beyond, 2012, S. 127; M. Wendel, Permeabilität im europäischen Verfassungsrecht, 2011, S. 573 ff.; M. Walter, Integrationsgrenze Verfassungsidentität, ZaöRV 2012, 177 (195 ff.).

93 BVerfG, 2 BvR 2728/13, Beschl. v. 14.1.2014 - OMT, Rn. 27.

94 BVerfG, 2 BvR 2728/13, Beschl. v. 14.1.2014 - OMT, Rn. 29. 
setzes das OMT-Programm in Deutschland verfassungswidrig wäre. Dies würde erklären, warum die Identitätskontrolle in der Vorlageentscheidung überhaupt gegen Ende des Beschlusses noch zur Sprache kommt. Die Senatsmehrheit ist der Ansicht, dass die Budgethoheit des Bundestages Teil der deutschen Verfassungsidentität ist und hält es offenbar für möglich, dass diese sowie die haushaltspolitische Gesamtverantwortung des Bundestages im Hinblick auf mögliche Verluste der Bundesbank irgendwie durch das OMT-Programm beeinträchtigt werden können. Wörtlich:

„Eine Verletzung der Verfassungsidentität des Grundgesetzes durch den OMTBeschluss käme in Betracht, wenn hierdurch ein Mechanismus begründet würde, der auf eine Haftungsübernahme für Willensentscheidungen Dritter mit schwerkalkulierbaren Folgewirkungen hinausliefe (vgl. BVerfGE 129, $124<179$ ff. >), so dass aufgrund dieses Mechanismus der Deutsche Bundestag nicht „Herr seiner Beschlüsse " bliebe und sein Budgetrecht nicht mehr in eigener Verantwortung ausüben könnte (vgl. BverfGE [sic!] 129, $124<177>$; 132, $195<239>$ ). "95

Und weiter:

„Ob sich darüber hinaus durch einzelne Umsetzungsmaßnahmen des OMT-Beschlusses im Hinblick auf mögliche Verluste der Bundesbank und sich daraus ergebende Folgewirkungen auf den Bundeshaushalt Auswirkungen auf das Budgetrecht des Deutschen Bundestages in einer Art. 79 Abs. 3 GG berührenden Weise ergeben können, ist gegenwärtig nicht absehbar. "96

Das Argument scheint also zu sein, dass das OMT-Programm zu erheblichen Verlusten der Bundesbank führen könnte, die dann den Bundeshaushalt lähmen könnten. Hierdurch würden die Wahlen zum Bundestag für die deutschen Wähler bedeutungslos, da ein in Fesseln geschlagener Haushalt dem Bundestag jeglichen Bewegungsspielraum nehmen würde. Dies würde wiederum die Demokratie in Deutschland in ihrem Kern betreffen, sodass das OMT-Programm letztlich die deutsche Verfassungsidentität beeinträchtigen würde.

Falls man jemals Bedarf für ein Beispiel eines weit hergeholten juristischen Arguments haben sollte - dies ist ein Beispiel. Die Argumentation erscheint fast zu fernliegend, um sie ernsthaft zu diskutieren. Die Bundesbank hat in ihrer Geschichte auf Grund von Abschreibungen auf Währungsreserven ${ }^{97}$ wiederholt Verluste eingefahren, ohne dass dies zum Demokratieproblem gemacht worden wäre. Eine Ausgleichspflicht gegenüber der Bundesbank aus dem Bundeshaushalt besteht nach deutschem Recht gar nicht. ${ }^{98}$ Es entbehrt nicht einer gewissen Ironie, dass eine

95 BVerfG, 2 BvR 2728/13, Beschl. v. 14.1.2014 - OMT, Rn. 102.

96 Dies - so das BVerfG - ist der Artikel, der die deutsche Verfassungsidentität definiert. BVerfG, 2 BvR 2728/13, Beschl. v. 14.1.2014-OMT, Rn. 103.

97 Abwertung des Dollars gegenüber starker DM.

98 Deutsche Bundesbank, Stellungnahme gegenüber dem Bundesverfassungsgericht zu den Verfahren 2 BvR 1390/12, 2 BvR 1421/12, 2 BvR 1439/12, 2 BvR 1824/12, 2 BvE 6/12 v. 21.12.2012, S. 28. 
Verpflichtung auf Ausgleich von nationalen Zentralbankverlusten sich am ehesten noch aus EZB-Vorgaben ergeben könnte, wie die Bundesbank andeutet. ${ }^{99}$ Freilich dringt die EZB auf einen solchen Ausgleich unter dem Aspekt der finanziellen Unabhängigkeit der nationalen Zentralbanken, es geht nicht um katastrophische Dimensionen einer Wertlosigkeit von auf dem Sekundärmarkt auf Veranlassung der EZB aufgekauften Staatsanleihen. Aber selbst wenn man eine Ausgleichspflicht aus dem Bundeshaushalt einmal unterstellt, dann erscheint eine Größenordnung, die den Bundeshaushalt auf lange Jahre lähmt und eine Wahlentscheidung irrelevant macht, reichlich fernliegend. Jedenfalls bleibt, dass es auch in einem hypothetischen Katastrophenverlauf in einer Wahlentscheidung immer noch um Alternativen ginge, wie mit dem Verlauf umzugehen ist.

Alarmierend bleibt jedenfalls eine prozessuale Anmerkung der sechs Mehrheitsrichter in diesem Zusammenhang:

„, Gegebenenfalls hätte der Senat dies auf der Grundlage der Auslegung des OMT-Beschlusses durch den Gerichtshof ohne erneute Vorlage zu prüfen und die Unanwendbarkeit entsprechender Umsetzungsmaßnahmen für den Geltungsbereich des Grundgesetzes festzustellen, weil die Identitätskontrolle keine Prüfung am Maßstab des Unionsrechts, sondern ausschließlich am Maßstab des deutschen Verfassungsrechts darstellt. "100

Hier offenbart sich deutlich das Missverständnis der Funktion des Verfassungsidentitätskonzeptes der Mehrheitsrichter: Sie akzeptieren ganz offenkundig nicht, dass im Kontext der Verfassungsidentität ein Vorabentscheidungsgesuch an den EuGH zwingend erforderlich ist, um sicherzustellen, dass die sich stellende Rechtsfrage überhaupt unter Art. 4 Abs. 2 AEUV fällt. Eine Vorlage durch ein nationales Gericht im Kontext der Identitätskontrolle wäre vorrangig auf die Auslegung von Art. 4 Abs. 2 AEUV durch den EuGH gerichtet. Die Senatsmehrheit scheint auf einer unilateralen, aus nationaler Sicht vorgenommenen Determinierung der Bedeutung von Art. 4 Abs. 2 AEUV zu bestehen.

Was dabei verkannt wird: Die Operationalisierung des Konzeptes „,nationale Identität" und ,nationale Verfassungsidentität“ als Schranke für das Europarecht ist auBerordentlich bedrohlich für die Rechtseinheit in der EU. Es besteht zudem ein enormes Missbrauchspotenzial auf nationaler Ebene, weil alles Mögliche an unangenehmen Verpflichtungen des Unionsrechts - von der Beihilfenkontrolle bis zur Freizügigkeit - mit dem unilateralen, unüberprüfbaren Hinweis auf die angeblich berührte nationale Verfassungsidentität ausgeschaltet werden kann. ${ }^{101}$

99 Ebd., die Bundesbank verweist auf die Konvergenzberichte der EZB und entsprechende Anforderungen, s. etwa EZB Konvergenzbericht Mai 2012, S. 28 ff., Konvergenzbericht Juni 2013, S. 33 ff.

100 BVerfG, 2 BvR 2728/13, Beschl. v. 14.1.2014 - OMT, Rn. 103.

101 Ein abwegig anmutendes Beispiel, das jedoch der Logik der einseitigen Identitätsdefinition folgt: Als nächstes könnte irgendein deutsches Gericht Volkswagen oder Opel zum Teil der deutschen Verfassungsidentität erklären und so Subventionen für traditionelle deutsche Schlüsselindustrien der europäischen Beihilfekontrolle entziehen. 
Verfassungsidentität als Schranke kann nur in einem echten Kooperationsverhältnis zwischen den Gerichten funktionieren. Im Hinblick auf die aktuelle Vorlageentscheidung des BVerfG stellt sich jedoch die Frage, ob die Motivation für die Erwähnung der Verfassungsidentität nicht die Orientierung am genauen Gegenteil nahe legt.

\section{Widersprüchlichkeiten, Kontinuitätsfehler und logische Sprünge in der Europaerzählung des BVerfG}

Aus analytischer Sicht ist das auffallendste Merkmal der allerersten Vorlage des BVerfG an den EuGH die Vielzahl der Widersprüchlichkeiten und Brüche, die sich in der Entscheidung finden. Die Sondervoten legen die meisten davon bereits offen. Im Einzelnen:

\section{Wahrnehmungsfehler}

Ein allgemeiner Test für die Orientierung eines europarechtsbezogenen nationalen Gerichtsurteils ist es, ob die Übernahme der Begründung durch alle anderen nationalen Gerichte in der EU denkbar wäre. Dies ist hier im Hinblick auf die Ultra vires-Kontrolle des BVerfG ersichtlich nicht der Fall. Wenn alle anderen Verfassungs- und Höchstgerichte in der EU die gleiche Argumentation verfolgten, dann zerstörte dies die Einheit des Europarechts und die Autorität des EuGH. Das Unionsrecht drohte zu einer Beliebigkeits- und Präferenzordnung zu mutieren, in der sich nationale Gerichte vorteilhafte Elemente aussuchen und alles andere beiseite lassen können.

Die Senatsmehrheit sieht dies nicht und versucht, ihre Position als Standpunkt zu präsentieren, den viele andere nationale Gerichte in der EU teilen und bestärkt zugleich andere Gerichte darin, dieser Sicht zu folgen. Hier bestehen mindestens zwei Wahrnehmungsfehler.

Zum einen wird die Gefahr für Rechtseinheit und Rechtsgemeinschaft aus ansteigenden Zahlen nationaler Gerichte, die sich als Letztentscheider über das Unionsrecht verstehen, nicht thematisiert und damit ganz offenbar unterschätzt. Darüber hinaus besteht aber auch ein Wahrnehmungsfehler der Senatsmehrheit im Hinblick auf die Größe der Gefolgschaft, die es hinsichtlich der Ultra vires-Kontrolle hat. Die Richter der Senatsmehrheit nennen in ihrer Vorlageentscheidung ${ }^{102}$ Fälle aus Dänemark, Estland, Frankreich, Irland, Italien, Lettland, Polen, Schweden, Spanien und der Tschechischen Republik und implizieren damit, dass die deutsche Sichtweise des BVerfG einen breiten Konsens abbildet. Dies ist so nicht richtig. 10 von 28 mitgliedsstaatlichen Rechtsordnungen wären noch nicht einmal eine Mehrheit. Vor allem aber vermengt die Senatsmehrheit unterschiedlichste Arten von Entscheidungen, die ganz verschiedenen Motivationen und Argumenten entspringen 
und in einen jeweiligen nationalen Kontext einzubetten sind. Allein der Hinweis auf das tschechische Landtova-Urteil hält einer näheren Analyse insofern stand, als dort tatsächlich das bundesverfassungsgerichtliche Ultra vires-Konzept positiv rezipiert und operationalisiert wird. Im Hintergrund steht indessen ein spezifischer nationaler Disput tschechischer Höchstgerichte. ${ }^{103}$ Alle anderen der vom BVerfG erwähnten nationalen Gerichte bleiben im Bereich von prinzipiellen Erwägungen, hypothetischen Verläufen und Theorie.

Es bleibt allerdings der Eindruck, dass die Art und Weise wie die Mehrheitsrichter angebliche Verbündete unter den anderen mitgliedsstaatlichen Höchstgerichten suchen und präsentieren, nicht recht zu einer Kooperations- und Dialogrhetorik passen will, wie sie bei anderen Gelegenheiten gepflegt wird. Vielleicht geht es manchen doch mehr um Frontlinien gegen den EuGH als um Kooperation.

\section{Die Verwechslung von Recht und Politik - „imperial overstretch“?}

Das Kernargument in Sondervotum Lübbe-Wolff ist der Vorwurf einer Überschreitung der Grenzen des ,durch ein Gericht Entscheidbaren“ durch die Senatsmehrheit, welche ein Rechtsregime dort anzuwenden sucht, wo dies schlicht nicht passt. ${ }^{104}$ Letztlich führt dies auf die Grenzlinie zwischen Recht und Politik. Das BVerfG hat sich von Anbeginn in der Euro-Krise intensiv eingemischt, mehr als jedes andere Höchstgericht in der EU. Auch wenn das BVerfG formal nur auf Anträge und Klagen reagiert hat und darauf beharren wird, dass seine Argumentation ausschließlich rechtlicher Natur sei, so hat es doch mit seinen Entscheidungen im In- und Ausland bewusst in eine komplexe und hochpolitische Debatte hineingewirkt.

Der Vorwurf eines richterlichen Aktivismus, einer zu starken Einmischung und zu politischen Argumentation ist für ein Verfassungsgericht freilich nichts Neues. Er kehrt regelmäßig wieder. Wenigstens zwei Punkte weisen im vorliegenden Fall allerdings darauf hin, dass vielleicht doch Grenzen überschritten werden.

Zunächst geht es dabei um die konkrete Rolle, die das BVerfG in der Euro-Krise eingenommen hat. Das BVerfG steht unter einer weltweiten Beobachtung durch politische und ökonomische Akteure hinsichtlich der Entscheidungen, die für die Euro-Krise von Relevanz sind. Dem BVerfG ist dabei wiederholt die Macht zugewachsen, mit einem Urteil die Weltwirtschaft aus den Angeln zu heben. Wäre das Gericht beispielsweise den Argumentationslinien der Antragsteller in den Verfahren um den EFSF oder den ESM (die beiden Rettungsschirme) gefolgt, etwa durch ein Verbot der Mitwirkung der Bundesrepublik an diesen beiden Konstrukten, dann hätte dies unmittelbar unüberschaubare Verwerfungen an den Finanzmärkten verursacht. Kein Gericht sollte die Macht haben, die Weltwirtschaft in die Knie zu

103 S. hierzu J. Komarek, Playing With Matches: The Czech Constitutional Court's Ultra Vires Revolution, http:// www.verfassungsblog.de/de/playing-with-matches-the-czech-constitutional-courts-ultra-vires-revolution.

104 BVerfG, 2 BvR 2728/13, Beschl. v. 14.1.2014 - OMT, Sondervotum Lübbe-Wolff, Rn. 3-9. 
zwingen. Etwas läuft fundamental falsch, wenn sich ein einziges Gericht in einem einzigen Nationalstaat in einer solchen Rolle wiederfindet.

Das Sondervotum Lübbe-Wolff erinnert zutreffend an etablierte und erprobte Mechanismen, derer sich Gerichte in einer solchen Situation bedienen können. Zu nennen wäre etwa die ,political question“-Doktrin oder das Konzept richterlicher Selbstbeschränkung. ${ }^{105}$ Das BVerfG hat diese Mechanismen bislang in der EuroKrise nicht hinreichend deutlich aktiviert. ${ }^{106}$ Es bewegt sich mit der Vorlageentscheidung in Sachen OMT-Programm am weitesten von dieser Zurückhaltung weg: Das Gericht versucht sich als Mitspieler auf dem Spielfeld der Zentralbanken und Volkswirte. Dies wird nicht weiterführen, insbesondere wenn die Realität nicht als Argument zählt. In den Worten der Senatsmehrheit: „Auf die (ökonomische) Richtigkeit oder Plausibilität der Begründung des OMT-Beschlusses kommt es insofern nicht an."107 Beobachter kommentieren dies als „masterpiece of judicial arrogance". 108

Sinnvoll wäre es demgegenüber gewesen, klarzustellen, dass in der Euro-Krise die Grenzen dessen, was das Recht und die Juristen leisten können, nahezu erreicht sind und dass bestimmte Entscheidungen von denjenigen zu treffen sind, die demokratisch gewählt wurden und die gegebenenfalls zur Verantwortung gezogen werden können. Wenn ein nationales Parlament beschließt, sich der maßgeblichen unabhängigen Zentralbank nicht entgegen zu stellen, dann ist dies eine solche Entscheidung, dann soll es so sein. Es kann nicht die Aufgabe des BVerfG sein, direkt gewählten Parlamentariern vorzuschreiben, ,etwas“ zu tun, ohne selbst dieses ,etwas“ näher spezifizieren zu können. ${ }^{109}$

Zweitens ergeben sich auch im Hinblick auf die Art und Weise, wie einige Richter außergerichtlich agieren, Nachfragen zur Grenze zwischen Recht und Politik. Sicherlich kann es hilfreich sein, wenn Verfassungsrichter die Urteile des BVerfG erklären, um Missverständnisse zu vermeiden. Dem lässt sich freilich der alte Satz vom Richter, der durch seine Urteile spricht, entgegenhalten. Wenn Urteile nicht mehr aus sich selbst heraus verständlich sind und es beispielsweise einer neueren Praxis nach einleitender Bemerkungen bei der Urteilsverkündung bedarf, dann liegt das möglicherweise auch an den Urteilen. Einige Beobachter meinen darüber hinaus, dass wenn Richter des BVerfG mit dem Präsidenten der Europäischen Kommission so zusammentreffen, wie dies sonst politischen Akteuren vorbehalten

105 BVerfG, 2 BvR 2728/13, Beschl. v. 14.1.2014-OMT, Sondervotum Lübbe-Wolff, Rn. 4, 7, 9.

106 Zumindest vom Ergebnis her lässt sich ist immerhin BVerfGE 132, 195 - ESM und Fiskalvertrag (einstweiliger Rechtsschutz) als Beispiel für eine Vermeidung unabsehbarer Folgen diskutieren.

107 BVerfG, 2 BvR 2728/13, Beschl. v. 14.1.2014 - OMT, Rn. 98.

108 Ch. Secondat u.a. (Fn. 13).

109 BVerfG, 2 BvR 2728/13, Beschl. v. 14.1.2014-OMT, Sondervotum Lübbe-Wolff, Rn. 18, 22. 
ist, ${ }^{110}$ etwas aus dem Lot geraten ist. Ähnlich werden Auftritte von Bundesverfassungsrichtern vor der Bundespressekonferenz bewertet. ${ }^{111}$ Selbst diejenigen, die diese Kritik unberechtigt und überzogen finden - was in mindestens einem Fall zutrifft $^{112}$ - werden einräumen, dass die Art der Kritik über die übliche Kritik an aktiven Verfassungsrichtern hinausgeht und eine andere Dimension hat: Es steht der Vorwurf im Raum, dass Richter sich wie Politiker verhalten. ${ }^{113}$

Parallel zu dieser Entwicklung versuchen Politiker zunehmend, die Befindlichkeiten und Einschätzungen des BVerfG vorauszusehen und sich dementsprechend zu positionieren. Natürlich ist nichts dagegen einzuwenden, dass Politiker den durch die Verfassung vorgegebenen Rahmen zu respektieren suchen. Was in der EuroKrise passiert, geht jedoch ersichtlich darüber hinaus, sobald hier verfassungsrechtliche Argumente politische Argumente schlicht ersetzen. Die Bundesregierung Ministerialbürokratie wie Minister, Kanzlerin und Kanzleramt - ist an dieser Entwicklung nicht ganz unschuldig, spielt man doch in Deutschland wie auch in Verhandlungen in Brüssel immer wieder die „Karlsruhe-Karte“. Typischerweise wird dabei wie folgt auf einen Vorstoß reagiert, um diesen zu neutralisieren: „Das ist womöglich eine gute Idee, allerdings wird das BVerfG dies [einsetzen: Art. 114 AEUV als Rechtsgrundlage die Bankenabwicklung/ eine direkte Bankenrekapitalisierung durch den ESM/ uvm.] leider, leider voraussichtlich nicht gutheißen."114 Das Resultat dieser Entwicklung mutet in Teilen paradox an: Auf der einen Seite Politiker, die versuchen, nur noch wie Verfassungsjuristen zu denken und in verfassungsrechtlichen Bahnen argumentieren und dabei die politischen Parameter aus dem Blick verlieren. Auf der anderen Seite Verfassungsrichter, die in ihrem Auftreten wie Politiker wahrgenommen werden und politisch wirken. Nochmals: Gänzlich neu ist dies nicht, aber die Dimensionen könnten es sein.

\section{Die ,kühne“" Zulässigkeitsrechtsprechung}

Im Lissabon-Urteil hat das BVerfG die Möglichkeit einer Kodifizierung seiner Kreationen, der Ultra vires-Kontrolle und der Identitätskontrolle, als förmliche neue

110 S. hierzu: Visit of Andreas Voßkuhle, Chairman of the Federal Constitutional Court of Germany, to the European Commission: „José Manuel Barroso, President of the EC, and Maroš Šefčovič, Vice-President of the EC in charge of Inter-Institutional Relations and Administration, received Andreas Voßkuhle, Chairman of the Federal Constitutional Court of Germany. Discussions focused on how to best ensure the respect of democracy and the rule of law in the EU and its Member States."Verfügbar unter: http://ec.europa.eu/avservices/photo/ photoByReportage.cfm?ref $=023108 \&$ sitelang=en.

111 H. Müller Vogg, Voßkuhle spricht nicht nur durch seine Urteile, Bayernkurier, 9.3.2013; H. Wefing, Gefährlicher Flirt - Warum Verfassungsrichter Sicherheitsabstand zur Politik halten sollten, Die Zeit 11/2013 v. 7.3.2013.

112 Die Darstellung in Merkels Chef, Der Spiegel v. 4.3.2013, S. 20 ff. mischt belegte Aussagen mit willkürlich unterstellten Wertungen der Autoren, wie der letzte - unzutreffende - Satz des Beitrags illustriert.

113 Man denke nur an den Ausbruch des damaligen Innenministers Friedrich im Frühjahr 2013: „Wenn Verfassungsrichter Politik machen wollen, sollen sie bitte für den Deutschen Bundestag kandidieren.“, zit. nach $S$. Höll, Innenminister Friedrich rügt obersten Verfassungsrichter, Süddeutsche Zeitung v. 23.4.2013.

114 Näher zum Fallbeispiel Bankenaufsicht in diesem Kontext F. C. Mayer/D. Kollmeyer, Sinnlose Gesetzgebung? Die Europäische Bankenunion im Bundestag, DVBl. 2013, S. 1158. 
Rechtsbehelfe angesprochen. ${ }^{115}$ Der Gesetzgeber hat sich im Sommer 2009 mit dieser Möglichkeit befasst und sich dagegen entschieden. Dies hätte das BVerfG als Warnsignal dafür auffassen können, dass das , ursprünglich kühne“"116, im Maastricht-Urteil richterrechtlich geschaffene und im Lissabon-Urteil bestätigte Konstrukt einer Verfassungsbeschwerde aus einem Recht auf einen Bundestag, der noch etwas zu entscheiden hat (Art. 38 GG) auf wackeligen legitimatorischen Beinen steht. ${ }^{117}$ Nichtsdestotrotz hat das BVerfG die Kriterien für die Zulässigkeit von Verfassungsbeschwerden im Kontext der Europäischen Integration kontinuierlich gelockert. Es verwundert kaum, dass sich mehr als 40.000 Beschwerdeführer für eine Verfassungsbeschwerde gegen ESM und Fiskalvertrag fanden.

Bemerkenswerterweise weicht die Senatsmehrheit mit dem OMT-Vorlagebeschluss die Zulässigkeitsvoraussetzungen gegen die ausdrücklichen Warnungen der Sondervoten noch weiter auf. ${ }^{118}$ Danach kann so ziemlich jeder vor das BVerfG mit der Behauptung ziehen, ein europäischer Rechtsakt sei ultra vires. In den Worten des Sondervotums Gerhardt: „Hingegen wird mit der Zulassung einer auf die Behauptung einer Verletzung von Art. 38 Abs. 1 GG gestützten ultra-vires-Kontrolle die Tür zu einem allgemeinen Gesetzesvollziehungsanspruch geöffnet, den das Grundgesetz nicht kennt“, zu einer „Popularklage““. ${ }^{119}$ Richter Gerhardt weist zudem zu Recht auf einen möglichen Konflikt mit dem System der Rechtsbehelfe auf europäischer Ebene (Art. 263 AEUV) im Hinblick auf Klagen europäischer Institutionen hin. 120

Es ist schwer vorstellbar, dass diese Rechtsprechung des BVerfG zur Zulässigkeit von Verfassungsbeschwerden nicht als eine Einladung aufgefasst wird, dem Gericht alle möglichen europäischen Rechtsakte zur Überprüfung vorzulegen, die nicht genehm sind. Diese Entwicklung ist umso bemerkenswerter, als das BVerfG sich auf der anderen Seite regelmäßig über die allgemeine Arbeitsbelastung (mehr als 6.600 Fälle im Jahr 2013) beschwert und den Gesetzgeber aktiv dahingehend zu beeinflussen versucht, den Zugang zum Gericht zu erschweren. ${ }^{121}$ Ausweitung der Zulässigkeit von Europaklagen und Begrenzung des Zugangs zum Gericht im Übrigen - das will nicht recht zusammenpassen.

Richterin Lübbe-Wolff äußert sich hierzu in ihrer abweichenden Meinung wie folgt:

„,Die hinsichtlich der Verfassungsbeschwerde sonst ins Auge fallende Tendenz des nach Entlastung suchenden BVerfGs zu sorgfältiger Pflege und kontinuier-

115 BVerfGE 123, 267, 253-255 - Lissabon, Rn. 241. Zu diesen Vorschlägen M. Wendel, Permeabilität im europäischen Verfassungsrecht, 2011, S. $479 \mathrm{ff}$.

116 BVerfG, 2 BvR 2728/13, Beschl. v. 14.1.2014 - OMT, Sondervotum Lübbe-Wolff, Rn. 15.

117 Zur Kritik s. etwa C. Tomuschat, Anmerkung, DVB1. 2014, S. 645 (646).

118 Kritisch hierzu BVerfG, 2 BvR 2728/13, Beschl. v. 14.1.2014 - OMT, Sondervotum Lübbe-Wolff, Rn. 16.

119 BVerfG, 2 BvR 2728/13, Beschl. v. 14.1.2014 - OMT, Sondervotum Gerhardt, Rn. 6, 7.

120 Ebd.

121 Näher hierzu: Karlsruhe klagt über gestiegene Arbeitsbelastung, FAZ v. 14.2.2014, S. 4. Natürlich sind 40.000 Antragsteller nicht gleichbedeutend mit 40.000 Fällen. Die Frage nach einer sinnvollen Verwendung der Gerichtsressourcen stellt sich aber trotzdem. Statistische Details sind verfügbar unter: http://www.bundesverfassungsgericht.de/organisation/statistik_2013.html. 
lichem Ausbau von Zulässigkeitshürden ist zwar auf dem Feld der europäischen Integration generell nicht zu beobachten. So weit wie im vorliegenden Fall hatte der Senat aber seine Befassungsbereitschaft bisher nicht ausgedehnt. "122

Diese Bemerkung ist selbsterklärend. Offensichtlich geht es der Senatsmehrheit darum, in europarechtlichen Fragen die Kontrolle und die Zugriffsmöglichkeiten zu behalten - um fast jeden Preis, jedenfalls um den Preis einer de facto-Popularklage. Sicherlich kann man um den richtigen Einsatz der begrenzten Ressource Rechtsprechung streiten. Die Entscheidung über die Schwerpunktsetzung, vielleicht auch über die Ausweitung der Ressource, obliegt indessen der Politik.

\section{Das Ultra vires-Paradoxon und der Honeywell-Widerspruch}

Es ist falsch, das OMT-Programm der EZB für kompetenzwidrig zu erklären (vgl. bereits oben, FAQs). Bei dem Programm handelt es sich nicht um einen Ultra vires-Akt. Selbst wenn man annähme, dass das Programm die Befugnisse der EZB überschreiten würde, so setzte sich das BVerfG doch in Widerspruch zu seiner eigenen Honeywell-Rechtsprechung, in der Kriterien für die Annahme eines Ultra vires-Aktes ausformuliert worden sind. ${ }^{123}$ Danach muss die Kompetenzüberschreitung offensichtlich sein und zu einer strukturell bedeutsamen Verschiebung des Kompetenzgefüges zulasten der Mitgliedstaaten führen. ${ }^{124}$

Beide Bedingungen sind vorliegend nicht erfüllt. Die Senatsmehrheit benutzt hier einen Trick, ${ }^{125}$ indem sie lediglich überprüft, ob Wirtschaftspolitik offensichtlich außerhalb der EU-Kompetenzen liegt, wohingegen der Test eigentlich dahingehend lauten müsste, ob der in Rede stehende europäische Akt überhaupt als Wirtschaftspolitik zu qualifizieren ist. Ohnehin ist die Frage, wie eine Kompetenzüberschreitung denn offenkundig sein soll, wenn das BVerfG etliche Monate für die entsprechende Feststellung benötigt, ${ }^{126}$ die beiden dienstältesten Richter anderer Auffassung sind und sich sogar Wirtschaftswissenschaftler und Zentralbankspezialisten nicht einigen können. Wer unter diesen Bedingungen von Offenkundigkeit spricht, kann auf das Kriterium auch gleich verzichten.

Darüber hinaus ist fraglich, wie ein angeblicher Ultra vires-Akt das Kompetenzgefüge in einem Bereich in einer strukturellen Größenordnung verschieben kann, in dem es gar keine nationalen Kompetenzen gibt. Den Mitgliedstaaten wird nichts genommen, da es schlicht keine nationale Institution gibt, die die Handlungen vornehmen könnte, die die EZB vornimmt.

BVerfGE 126, 286 - Honeywell, Leitsatz 1: „Das setzt voraus, dass das kompetenzwidrige Handeln der Unionsgewalt offensichtlich ist und der angegriffene Akt im Kompetenzgefüge zu einer strukturell bedeutsamen Verschiebung zulasten der Mitgliedstaaten führt.“ (Hervorhebung hinzugefügt).

125 BVerfG, 2 BvR 2728/13, Beschl. v. 14.1.2014 - OMT, Rn. 37.

126 BVerfG, 2 BvR 2728/13, Beschl. v. 14.1.2014 - OMT, Sondervotum Gerhardt, Rn. 16, 17. 
Ein weiteres Element gibt Anlass zu Zweifeln am Fortbestand der Honeywell-Kriterien. Zur Honeywell-Entscheidung gab seinerzeit Richter Landau ein Sondervotum ab, in dem er eine Abweichung der damaligen Senatsmehrheit vom LissabonUrteil durch die Einführung von ,überspannt[en] [...] Anforderungen an die Feststellung eines Ultra vires-Handelns" 127 beanstandete, die Ultra vires-Kontrolle bestehe „nur noch auf dem Papier". ${ }^{128}$ Im vorliegenden Verfahren gehörte Richter Landau zur Senatsmehrheit und sah offenkundig keinen Anlass, auf seine abweichende Meinung aus Honeywell zurückzukommen. Entweder hat Landau seine Meinung geändert und sich mit dem zuvor beklagten Standard der Ultra vires-Kontrolle nach Honeywell abgefunden. Oder der im Honeywell-Urteil formulierte Standard ist nicht mehr maßgeblich, so dass die Senatsmehrheit in Ultra vires-Fragen nun Landau folgt, der nicht davor ,zurückschreckt ${ }^{6129}$, einen Akt für ultra vires zu erklären. Problematisch daran erscheint, dass Begrifflichkeiten wie ,zurückschrecken“ oder plakative Slogans wie „Nicht nur Zähne zeigen - beißen!“"130 Ausdruck eines Konfrontationskurses sind, der die europäische Rechtsgemeinschaft in Mitleidenschaft ziehen kann.

Eine weitere Neuerung in der Ultra vires-Rechtsprechung des BVerfG benennt das Sondervotum Gerhardt. Gerhardt betont, dass die Senatsmehrheit die Ultra viresKontrolle selbst dann anwenden will, wenn keine Grundrechts- oder Verfassungsidentitätselemente in Frage stehen und sieht darin eine Ausweitung und Abkehr im Vergleich zur bisherigen Rechtsprechung. ${ }^{131}$

Allgemeiner betrachtet ist das Problem, dass Ultra vires-Konflikte um die Frage, wem das Letztentscheidungsrecht über Kompetenzen in einem Mehrebenensystem zusteht, letzten Endes nicht durch das Recht gelöst werden können. ${ }^{132}$ Verfassungsvergleichung bestätigt diesen Befund. ${ }^{133}$ Deswegen spricht vieles dafür, dass die Beanspruchung eines Ultra vires-Kontrollrechts vor allem dann einen stabilisierenden Effekt für die Kompetenzsituation entwickeln kann, wenn es bei der bloßen Möglichkeit einer solchen Kontrolle verbleibt. Sobald die Kontrolle aktiviert wird, können beide Seiten nur verlieren, weil es eben keine rechtliche Auflösung gibt. Der Präsident des BVerfG, Voßkuhle, formuliert dazu u.a. im Kontext der Ultra

127 BVerfGE 126, 286 - Honeywell, Sondervotum Landau, Rn. 95.

128 BVerfGE 126, 286 - Honeywell, Sondervotum Landau, Rn. 104.

129 Ebenda.

130 C. Hillgruber, Nicht nur Zähne zeigen - beißen!, NJW 2014, Heft 8, NJW-Aktuell, Editorial, S. 3. Die Dentalmetapher vom Zubeißen hat in regelmäßigen Abständen Konjunktur bei Hinweisgebern für Höchstgerichte, im englischen Sprachraum eher im Kontext von „Barks and Bites“ (Bellen und Beißen).

131 BVerfG, 2 BvR 2728/13, Beschl. v. 14.1.2014 - OMT, Sondervotum Gerhardt, Rn. 5.

132 Neu ist dies nicht: Alfred Verdross hat hierzu den Begriff der „Grenzorgane“ geprägt, Institutionen, die zwar rechtlichen Bindungen, aber keinerlei rechtlicher Kontrolle unterliegen, so dass die Lösung von Konflikten nur eine politische oder soziologische Frage sein kann, A. Verdross, Völkerrecht, 1950, S. 24, unter Verweis auf Hans Kelsen.

133 Es besteht eine aufschlussreiche Parallele in der US-amerikanischen Verfassungsgeschichte, im Hinblick auf Konflikte zwischen den einzelstaatlichen Obergerichten und dem US Supreme Court im 19. Jahrhundert. Auch hier ging es um die Frage, wer das Letztentscheidungsrecht über die Kompetenzen der übergreifenden politischen Ordnung hat, näher hierzu F. C. Mayer, Kompetenzüberschreitung (Fn. 38), S. 290-325. Ein Beispiel für diese gerichtlichen Konflikte ist Virginia Court of Appeals, Hunter's Lessee v. Martin, 4 Munf. 1 (1815). 
vires-Kontrolle: „,Notbremse-Verfahren“ behalten gerade dann ihre Berechtigung, wenn sie nicht in Anspruch genommen werden müssen. "134 Voßkuhle entwickelt in diesem Zusammenhang das Konzept eines ,Europäischen Verfassungsgerichtsverbunds“, bei dem es im Kern um Dialog, Kooperation, Offenheit und Harmonie zwischen dem EuGH und den nationalen Gerichten geht. Ob die Senatsmehrheit sich diesen Ansatz zu eigen macht, wird nicht zuletzt der Fortgang des OMT-Verfahrens erkennen lassen.

Jedenfalls entbehrt es nicht einer gewissen Ironie - wie das Sondervotum LübbeWolff betont - dass das BVerfG andere Institutionen mit einem Ultra vires-Vorwurf überzieht, während es zugleich selbst die Grenzen seiner eigenen Kompetenzen großzügig handhabt und die Grenzen seiner Expertise überschreitet, damit letztlich selbst ultra vires handelt. Dieser Befund lässt sich in rechtliche Kategorien übersetzen, nämlich als Möglichkeit eines Vertragsverletzungsverfahrens gegen Deutschland wegen der europarechtliche Grenzen überschreitenden Ultra viresAnsprüche des BVerfG.

\section{Das Demokratie-Paradoxon}

Aus einer demokratietheoretischen Perspektive stellen Verfassungsgerichte ein konzeptuelles Dauerproblem dar. Die Tatsache, dass einige wenige ernannte Richter den vielen direkt gewählten Abgeordneten Einhalt gebieten können, ist demokratietheoretisch nicht einfach zu erklären. Dies macht das Verhältnis zwischen dem Parlament und einem zur Normenkontrolle befugten Verfassungsgericht grundsätzlich zu einem speziellen Verhältnis. Sobald es um die Europäische Integration geht, ist das Verhältnis zwischen dem BVerfG und dem Bundestag nicht nur speziell, sondern kompliziert.

Einerseits stärkt das BVerfG seit einiger Zeit kontinuierlich die Rolle des Bundestags im Zusammenhang mit der Europäischen Integration gegenüber einer hinhaltenden Widerstand leistenden Bundesregierung, die es vorzieht, den Bundestag auf einer bestimmten Distanz zu halten, um selbst mehr Spielraum in Brüssel zu haben. Das Kernargument des Gerichts ist dabei das Demokratieprinzip: Nur wenn der Bundestag das Handeln der Bundesregierung im Unionskontext kontrollieren kann, ist die demokratische Legitimation eines nachfolgenden EU-Rechtsakts gewährleistet. Diese demokratische Legitimation ist ein Imperativ des deutschen Verfassungsrechts. Die Gleichung lautet „mehr Kontrolle durch den Bundestag gleich mehr Demokratie".

Im Prinzip ist nichts dagegen einzuwenden, wenn die nationalen Parlamente in EUAngelegenheiten gestärkt werden. Ihre Einbeziehung stellt sicher, dass es von der Wählerschaft aus eine Rückkopplung im Hinblick auf Entscheidungen auf Unionsebene gibt - nicht zuletzt weil Abgeordnete vielfach ganz konkret in Wahlkreisen 
Wählern begegnen, ihnen europäische Entwicklungen erklären und Rechenschaft ablegen müssen, was für Regierung und Ministerialbürokratie so nicht der Fall ist. Dennoch ergeben sich mindestens drei Probleme aus dem Ansatz des BVerfG, der primär über die Einwirkungsmöglichkeiten des Bundestags Demokratie in EU-Angelegenheiten sicherzustellen sucht.

Erstens spielt die Möglichkeit, dass demokratische Legitimation auch durch das Europäische Parlament hergestellt werden könnte, in der Vorstellung des BVerfG von europäischer Demokratie keine große Rolle. Die jüngeren Entscheidungen des BVerfG zum deutschen EP-Wahlrecht wurden verbreitet so wahrgenommen, dass Karlsruhe das Europäische Parlament in seiner Bedeutung herabstuft. ${ }^{135}$ In der Welt des Zweiten Senats kann Demokratie offenbar allein von den Völkern der Nationalstaaten ausgehen. Die Kritik im Schrifttum an dieser sehr deutschen Sicht einer „bundesrepublikanischen Volksdemokratie“136 wächst seit der Maastricht-Entscheidung von 1993 kontinuierlich an. Das Gericht bleibt davon bisher unbeeindruckt. Warum es einerseits das EP - gegen den Vertragswortlaut ${ }^{137}$ - weiter als Vertretung der „Völker“ sieht, andererseits aber das Argument, der Wegfall des Sperrklauseln in Deutschland schwäche den Einfluss Deutschlands im EP nicht hört, erklärt das BVerfG übrigens nicht.

Zweitens besteht ein Abstand zwischen dem hohen bundesverfassungsgerichtlichen Anspruch in Sachen Europatauglichkeit des Parlaments und der Realität. Zweifelsohne hat der Bundestag in den letzten Jahren beachtliche Fortschritte in Sachen Europatauglichkeit gemacht. Gleichwohl befand das BVerfG wiederholt, dass es den Abgeordneten nicht gelungen war, den hohen Anforderungen des BVerfG gerecht zu werden. Die Kritik des BVerfG am Bundestag ist dabei in manchen Fällen sehr, ${ }^{138}$ in anderen Fällen weniger berechtigt. Die Wahrnehmung der Parlamentarier ist dabei nicht selten, dass sie bevormundet werden und dass das BVerfG sich von einem Idealbild des Parlaments leiten lässt, das so in der politischen Realität nicht

135 Es lässt sich zwar darüber diskutieren, dass dies gar nicht die Absicht des BVerfG ist, wie einige Richter außerhalb von Entscheidungen wiederholen, aber es ist noch immer die Wahrnehmung, s. etwa das jüngste Urt. v. 26.2.2014, 2 BvE 2/13 u.a. - Drei-Prozent-Sperrklausel, und das Medienecho hierauf.

136 S. etwa B.-O. Bryde, Die bundesrepublikanische Volksdemokratie als Irrweg der Demokratietheorie, Staatswissenschaften und Staatspraxis 1994, S. 305; J.H.H. Weiler, The State "über alles". Demos, Telos and the German Maastricht Decision, FS Everling, 1995, Bd. 2, S. 1651. Zu einer jüngeren Analyse s. C. Franzius, Demokratisierung der Europäischen Union, EuR 2013, S. 655.

137 Nach Art. 14 Abs 2 EUV setzt sich das EP aus Vertretern der Unionsbürger zusammen.

138 BVerfGE 130, 318 - Neuner-Gremium. 
besteht und nicht bestehen kann. ${ }^{139}$ Die Ironie dabei ist, dass der demokratisch legitimierte Bundestag im Namen der Demokratie Vorgaben vom BVerfG als einem Gremium erhält, das selbst weder unmittelbar gewählt noch rechenschaftspflichtig ist. Man kann sich gelegentlich fragen, ob die Vorstellungen, die im BVerfG vom Parlament und seiner Arbeitsweise herrschen nicht von einer zu romantischen Vorstellung von einem Kamingespräch-Parlament geprägt sind, wo ehrenwerte Männer und Frauen in einem aufgeklärten Diskurs um den rechten Weg des Gemeinwesens ringen und sich dabei alle erforderliche Zeit nehmen können, um zu deliberieren und die beste Lösung zu finden. Innerhalb des von der Verfassung vorgegebenen Rahmens natürlich. Dies entspricht indessen schlicht nicht der Realität des deutschen Parlamentarismus im 21. Jahrhundert - ein Parlamentarismus, der sich in einer zunehmend beschleunigten Gesellschaft in einem Land von rund 80 Millionen Einwohnern gegenüber den Auswirkungen der Globalisierung und einer weltweiten Interdependenz behaupten muss. Die Zeit, die man sich in Karlsruhe einfach nehmen kann, besteht in Berlin so meist nicht. ${ }^{140}$

Drittens schließlich stellt sich die Frage, ob nicht Grenzen für die Aufwertung von Rechten des Parlaments in Sicht kommen. Es erscheint wenig sinnvoll, ein Parlament mit Aufgaben und Funktionen zu betrauen, die die Exekutive schlicht besser übernehmen kann. Wenn sich dringliche Fragen stellen und Entscheidungen innerhalb weniger Stunden zu treffen sind, dann ist es nahezu unmöglich, kurzfristig das Plenum des Bundestages mit seinen mehr als 600 Parlamentariern einzuberufen. ${ }^{141}$ Wenn in Verhandlungen eine gewisse Beweglichkeit der Verhandlungspositionen erforderlich ist, dann wird es nicht weiter führen, wenn jede einzelne Entwicklung einer laufenden Verhandlung durch das Plenum des Bundestags bestätigt werden muss. Ganz abgesehen davon bestehen Situationen, in denen die Stärkung des Parlaments keine sinnvolle Option darstellt. Dies ist beispielsweise im Hinblick auf das Handeln der EZB der Fall: Eine unabhängige Zentralbank agiert schon per

139 Hier spielen einige eher technische Fragen eine Rolle, z.B. die Tatsache, dass das BVerfG wiederholt mündliche Verhandlungen, bei denen die Anwesenheit von Abgeordneten mehr oder weniger erwartet wurde, an Tagen terminiert hat, an denen wichtige Plenardebatten anstanden. Einige außerhalb von Entscheidungen liegende Vorgänge werden von manchen Beobachtern und Parlamentariern als paradigmatisch angesehen, so etwa die öffentliche Diskussion zwischen dem Präsidenten den BVerfG und dem Bundestagspräsidenten Anfang 2013, wo der Richter das Verhältnis zwischen BVerfG und Gesetzgeber in EU-Angelegenheiten als ein „"Fördern und fordern" beschrieb, einem Slogan, der mit dem eher kontroversen Hartz IV-Programm in Verbindung gebracht wird. Beim selben Anlass äußerte der Präsident des BVerfG, der offenbar etwas Freundliches über den Bundestagspräsidenten sagen wollte: „Ich würde ihn dafür ja fast adoptieren, wenn er nicht Soziologe wäre“. S. dazu R. Roßmann, Spitzen der Präsidenten, Süddeutsche Zeitung v. 1.3.2013. Zu betonen ist dabei, dass es auch unfaire, das BVerfG unsachlich angehende Wendungen und Elemente in diesen Berichten gibt, s. insofern die Analyse der medialen Berichterstattung von K. Gelinsky, Voßkuhle und die Presse: Stimmungsumschwung oder Manipulation?, abrufbar unter: http://www.verfassungsblog.de/de/voskuhle-und-die-pressestimmungsumschwung-oder-manipulation.

140 S. dazu die Ansicht des Präsidenten des BVerfG, Voßkuhle: ,Das Bundesverfassungsgericht ist tatsächlich einer der ganz wenigen Orte, an denen man abgeschirmt von äußeren Einflüssen Entscheidungen so intensiv durchdenken kann, wie man es für erforderlich hält. Es hat mitunter fast etwas Klösterliches. Das heißt nicht, dass wir uns nicht beeilen, wir nehmen uns aber gerade in den großen Verfahren die Zeit, die wir brauchen.", Organisierter Widerstand (Interview), Die Zeit v. 16.5.2012, S. 8.

141 Dies war Teil der Frage, als der Bundestag Aufsichtsbefugnisse in der Euro-Rettung an ein opakes Komitee delegieren wollte, BVerfGE 130, 318 - Neuner-Gremium. 
Definition außerhalb der Reichweite parlamentarischer Einwirkung, ${ }^{142}$ andernfalls wäre sie nicht unabhängig. In den Worten des BVerfG: „Die Verselbständigung der meisten Aufgaben der Währungspolitik bei einer unabhängigen Zentralbank löst staatliche Hoheitsgewalt aus unmittelbarer staatlicher oder supranationaler parlamentarischer Verantwortlichkeit, um das Währungswesen dem Zugriff von Interessentengruppen und der an einer Wiederwahl interessierten politischen Mandatsträger zu entziehen"143.

Eine Zentralbank stellt genau besehen eine ähnliche Demokratie-Anomalie dar wie ein Verfassungsgericht - beide sind „,counter-majoritarian institutions“, deren Demokratiedefizite sich allein mit ihrer Funktion rechtfertigen lassen. Es entbehrt nicht einer gewissen Ironie, dass im OMT-Fall ausgerechnet zwei derartige Institutionen aneinander geraten, und dies auch noch im Namen der Demokratie.

$\mathrm{Zu}$ den Demokratiefragezeichen im vorliegenden Kontext gehört auch, dass die Logik des Vorlagebeschlusses darauf hinauslaufen könnte, dass sechs unabhängige, niemanden gegenüber rechenschaftspflichtige Richter den demokratisch gewählten und verantwortlichen Gesetzgeber anweisen, ,etwas zu tun“. Dabei ist nicht auszuschließen, dass die Richter der Senatsmehrheit für sich in Anspruch nehmen, einer Art schweigenden Mehrheit in Deutschland in Sachen Eurorettung eine Stimme zu verleihen, eine „Opposition gegenüber dem Mainstream“144. Aber bei Annahmen zu schweigenden Mehrheiten kann man sich leicht verschätzen, sie sollten jedenfalls nicht maßgeblich für Verfassungsgerichtsentscheidungen sein. In den Worten des Sondervotums Gerhardt:

,,Wenn - um beim Fall zu bleiben - die Bundesregierung das OMT-Programm billigt und in die Grundlagen ihres eigenen Handelns einbezieht und der Deutsche Bundestag all dies sehenden Auges - vor dem Hintergrund einer intensiven öffentlichen Debatte, nach Anhörung des Präsidenten der Europäischen Zentralbank und ausweislich der Auskunft eines Mitglieds des Haushaltsausschusses des Deutschen Bundestages in der mündlichen Verhandlung aufgrund Beobachtung und Bewertung des Handelns der Europäischen Zentralbank - hinnimmt, liegt darin die Ausübung seiner demokratischen Verantwortung. Der Bundestag hätte ohne weiteres auf politischem Wege - etwa durch eine Entschließung - den OMT-Beschluss missbilligen, gegebenenfalls auch eine Nichtigkeitsklage androhen, die Reaktion der Europäischen Zentralbank und der Finanzmärkte abwarten und dann weitere Konsequenzen ziehen können. Dass er all dies nicht getan hat, indiziert kein Demokratiedefizit, sondern ist Ausdruck einer Mehrheitsentschei-

142 Es gibt dennoch eine indirekte Verbindung zwischen dem Deutschen Bundestag und der Rechtssache OMT. Die EZB hat ihn dadurch geschaffen, dass der Zugang zum OMT-Programm nur den Mitgliedstaaten offen steht, die auch an einem ESM-Programm teilnehmen. Dort geschieht nichts ohne die Zustimmung des Bundestages. Folglich könnte das BVerfG das Parlament dazu anhalten, ESM-Programme nur dann zu billigen, wenn die EZB sich verpflichtet hat, für den fraglichen Mitgliedstaat bestimmte OMT-Grenzen einzuhalten.

143 BVerfGE 89, 155, 208 - Maastricht.

144 Richter Huber bei einem öffentlichen Auftritt an der Universität Jena am 20.6.2013. 
dung für eine bestimmte Politik zur Bewältigung der Staatsschuldenkrise im Euro-Währungsraum. "145

\section{Das Deutsche-Interessen-Paradox}

Es existiert eine Welt außerhalb Deutschlands und die Richter des BVerfG wissen darum. Anderenfalls würde man sich kaum die Mühe machen, bestimmte Entscheidungen zeitgleich oder zeitnah auch in englischer Übersetzung zu veröffentlichen. Gleichwohl erscheint die Perspektive der Senatsmehrheit gleich unter mehreren Aspekten als sehr deutsch.

Sie erscheint stark geprägt von einer „Die-gegen-uns“-Logik. Die wirtschaftlichen und sozialen Verwerfungen in anderen Mitgliedstaaten aufgrund der Eurokrise spielen in den Entscheidungen des BVerfG keine große Rolle. Gleiches gilt für wechselseitige wirtschaftliche Verflechtungen und Abhängigkeiten in der Eurozone oder die Tatsache, dass die deutsche Exportwirtschaft von der Liquidität bestimmter Mitgliedstaaten ,,im Süden“ profitiert hat. Wenig Platz besteht im Weltbild der Senatsmehrheit offenbar für ein Konzept wie Solidarität über die nationalstaatlichen Grenzen hinaus. Manche mögen hier sogar ein Defizit an verfassungsrechtlicher Empathie erkennen, wenn man bedenkt, dass einige der Konzepte, die das BVerfG ganz selbstverständlich für die deutsche Verfassungsordnung in Anspruch nimmt und verteidigt, zum Teil in der Euro-Krise erst fortentwickelt, wie etwa Selbstbestimmung, Haushaltsautonomie etc., nicht mehr allen Mitgliedstaaten der Eurozone zur Verfügung stehen. Was bleibt von der verfassungsrechtlichen Selbstbestimmung, der ,souveränen Staatlichkeit“, wie das BVerfG seit einiger Zeit formuliert, der Herrschaft über die Verträge, die dem BVerfG so wichtig ist, oder dem freien Willen eines Volkes in einem Mitgliedstaat, der sich in einem ESM-Programm mit seinen strengen ,Konditionalitäten“ unterwerfen muss? Von den möglichen Auswirkungen - vielleicht sogar Schäden -, die die Euro-Rechtsprechung des BVerfGs in anderen Mitgliedstaaten verursacht, ist schon gar keine Rede. Die Frage nach der Legitimität von Gerichtsentscheidungen eines deutschen Gerichts mit faktischen Durchgriffseffekten in andere Mitgliedstaaten hinein, ist kein Thema für das BVerfG. Es verharrt auf der Sondervotenebene. ${ }^{146}$

Das einfache Gegenargument aus Sicht des BVerfG liegt auf der Hand. Danach befinden sich all diese Fragen schlicht außerhalb der Zuständigkeit des BVerfG. Das BVerfG ist dafür zuständig, das Grundgesetz in Deutschland zu wahren. Und mag man auch außerhalb Deutschlands immer mehr eine neue Form deutscher Hegemonie wahrnehmen, eine Hegemonie durch Verfassungsgerichtsurteile, so würde das BVerfG demgegenüber sicherlich darauf bestehen, dass es allenfalls um Nebeneffekte geht, weil sein Blickwinkel allein nach innen gerichtet ist.

145 BVerfG, 2 BvR 2728/13, Beschl. v. 14.1.2014 - OMT, Sondervotum Gerhardt, Rn. 23.

146 S. dazu die Hinweise von Richterin Lübbe-Wolff, BVerfG, 2 BvR 2728/13, Beschl. v. 14.1.2014 - OMT, Sondervotum Lübbe-Wolff, Rn. 28. 
Genau darin liegt indessen wohl ein weiteres Problemelement. Sämtlichen EuroEntscheidungen des BVerfG liegt als durchgehendes Motiv mehr oder weniger explizit der Schutz deutscher Interessen zugrunde. Wenn das BVerfG verlangt, dass keine ESM-Maßnahme ohne Zustimmung des Bundestags getroffen werden darf, dann liegt dem die Annahme zugrunde, dass der Bundestag nicht gegen deutsche Interessen entscheiden wird. Aber was, wenn es gerade das deutsche nationale Interesse ist, dass das Handeln der Bundesrepublik nicht als in erster Linie nur egoistisch dem deutschen Interesse dienend wahrgenommen wird?

Eine mögliche Antwort auf diese Frage ist, dass genau aus diesem Grunde die Politik und nicht ein nationales Verfassungsgericht das letzte Wort in Angelegenheiten der Europäischen Integration haben muss. Schon aus systemimmanenten Gründen wird es für Institutionen wie das BVerfG stets vorrangig sein, sich auf den Schutz nationaler Belange zurückzuziehen. Für Deutschland ist diese Frage noch etwas komplexer, weil die Verfassungsgeber um das Deutsche-Interessen-Paradox wussten. Dies spiegelt sich in der Verfassungsentscheidung für die europäische und internationale Zusammenarbeit, wie sie bereits in der Präambel des GG 1949 sichtbar ist ${ }^{147}$ und die mit dem Deutschlandvertrag von $1952{ }^{148}$ Grundlage und Bedingung für die Wiedererlangung von (Teil-)Souveränität war. Dem GG ist es damit eigentlich schon gelungen, das Deutsche-Interessen-Paradox einzufangen: Es liegt im deutschen Interesse, dass das deutsche Interesse nicht als deutsches Interesse wahrgenommen wird, sondern als europäisches Interesse. Indem das BVerfG zwischenzeitlich ein verfassungsrechtliches Prinzip der Europarechtsfreundlichkeit ausdrücklich anerkannt hat, hat es dies in seiner Rechtsprechung ja eigentlich schon längst nachvollzogen. ${ }^{149}$ Diese Einsicht muss freilich dann auch in der jeweiligen verfassungsgerichtlichen Entscheidungspraxis immer wieder aktualisiert werden auch die Bezugnahme auf Art. 79 Abs. 3 GG und die Argumentation mit im Hinblick auf weitere Integrationsschritte angeblich nahende Grenzen des Grundgesetzes kann eine Eigendynamik entwickeln, die in argumentative Endstationen führt.

147 Um ein weiteres Beispiel für die Offenheit der Verfassung zu geben: Nach Art. 24 Abs. 3 GG ordnet sich das Grundgesetz ebenfalls einer allgemeinen, umfassenden und verpflichtenden Entscheidungsinstanz unter - ohne einen ultra-vires- oder einen Identitätsvorbehalt: „Zur Regelung zwischenstaatlicher Streitigkeiten wird der Bund Vereinbarungen über eine allgemeine, umfassende, obligatorische, internationale Schiedsgerichtsbarkeit beitreten.".

148 BGB1 1954 II S. 59

149 BVerfGE 123, 267 - Lissabon, Leitsatz 4. 


\title{
V. Zusammenfassung und Ergebnis
}

\author{
„, Warum machen wir das?“ \\ „Irgendetwas muss man doch machen. \\ Nicht wahr?" \\ (Rebel without a cause, 1955/56)
}

\begin{abstract}
„Dass einige unabhängige deutsche Richter unter Berufung auf die deutsche Auslegung des Demokratieprinzips und auf die Grenzen, die sich hieraus und aus unserer Lesart der Art. $123 \mathrm{ff}$. AEUV für die zulässigen Befugnisse der unabhängigen Europäischen Zentralbank ergeben, eine Entscheidung mit unkalkulierbar weitreichenden Konsequenzen für die ins Werk gesetzte Währung der gesamten Eurozone und die davon abhängigen Volkswirtschaften treffen, erscheint als Anomalie von höchst zweifelhafter demokratischer Qualität. "150
\end{abstract}

Dieser Folgerung im Sondervotum Lübbe-Wolff ist kaum etwas hinzuzufügen. Dass Grenz- und Ausnahmefälle kaum zur Regelbildung taugen - „Hard cases make bad law"151 - ist keine neue Erkenntnis. Auch Unstimmigkeiten und Widersprüchlichkeiten in einer Entscheidung lassen nicht auf, gutes Recht” schließen. Mit Blick auf die Sondervoten der zwei dienstältesten Richter des Senats stellt sich der OMTVorlagebeschluss des BVerfG insgesamt als eine schwache Entscheidung eines tief gespaltenen Senats dar. Das ist zu bedauern. Die historisch erste Vorlageentscheidung des BVerfG an den EuGH hätte ein besserer Fall sein sollen.

Im Hinblick auf die Sachfrage hätte der Zweite Senat so entscheiden sollen wie bereits 2011 im ersten Euro-Verfahren zur Griechenlandhilfe und zum temporären Rettungsschirm EFSF: Seinerzeit wurde mit dem Securities Market Programme (SMP) der EZB ein Vorläufer des OMT-Programms angegriffen. Das BVerfG erklärte entsprechende Verfassungsbeschwerden schlicht für unzulässig, da sie sich nicht gegen einen Akt deutscher öffentlicher Gewalt richteten. ${ }^{152}$

„Rebels without a cause“ oder doch ,with a cause“- welche Motive leiten eigentlich die Mehrheitsrichter? Kritiker unterstellen alles Mögliche an Motivationen, von der anti-europäischen Grundeinstellung bis hin zum richterlichen Ego. Auch hier führen allzu simple Antworten indessen nicht weiter. Ich vermag keine Anti-Europäer oder gar Europafeinde auf der Richterbank zu erkennen. Vieles spricht dafür, dass die Richter tatsächlich glauben, dass sie das Richtige tun. Sie sehen sich wohl wirklich als letzte Verteidigungslinie, als die letzte Bastion zum Schutz der Verfassung. Dabei ist der Weg Deutschlands in der Europäischen Integration kein Gegensatz zu dieser Verfassung - die Europäische Integration ist integraler Teil der Verfassung

150 BVerfG, 2 BvR 2728/13, Beschl. v. 14.1.2014 - OMT, Sondervotum Lübbe-Wolff, Rn. 28.

151 S. etwa Oliver Wendell Holmes in der US Supreme Court Entscheidung Northern Securities Co. v. United States, 193 U.S. 93 U.S. 197, 400-401 (1904), abweichende Meinung.

152 BVerfGE 129, 124, 175 f. - Griechenlandhilfe/EFSF. 
und des Vermächtnisses der Verfassungsgeber von 1949. Das BVerfG hat nicht das Monopol über diesen Weg zu bestimmen. Wenn dies nur vermittels einer Verfassungsänderung klarzustellen sein sollte, dann wäre eine solche dringlich.

Das BVerfG ist eine Institution, die der Bundesrepublik für mehr als 60 Jahre gute Dienste geleistet hat. Es hat einen maßgeblichen Beitrag dazu geleistet, in dem in jeder Hinsicht am Boden liegenden Nachkriegswestdeutschland eine Kultur der Demokratie, der Rechtsstaatlichkeit und des Grundrechtsschutzes zu etablieren. Es hat die Vorstellung einer in der Verfassung niedergelegten objektiven Wertordnung hochgehalten, unter besonderer Betonung der Menschenwürde. Nicht zuletzt hat es eine alte Weimarer Debatte um den Hüter der Verfassung zu einem Abschluss gebracht und unter Beweis gestellt, dass Carl Schmitt, der den Reichspräsidenten als Hüter der Verfassung sah, irrte, wenn er davor warnte, dass ein Verfassungsgericht letzten Endes immer Politik machen würde. ${ }^{153}$ Obsiegt hat hier vielmehr letztlich Hans Kelsen, der sich Schmitt in dieser Sache entgegenstellte und für ein Verfassungsgericht als Hüter der Verfassung aussprach. ${ }^{154}$

Hans Kelsen würde wahrscheinlich nicht sonderlich gefallen, wie das BVerfG in EU-Angelegenheiten agiert. Die Meinung der Senatsmehrheit, die den OMT-Beschluss trägt, steht für ein Verfassungsgericht, das sich in die falsche Richtung bewegt. Es ist eine Richtung, die das Risikopotenzial birgt, die Autorität des BVerfG, ${ }^{155}$ sein Vermächtnis und seine Leistungen im Bereich grundrechtsgestützter gesellschaftlicher Weiterentwicklung zu beschädigen, und die das BVerfG vom innerstaatlichen Grundrechtsschutz ablenkt, auf den es sich konzentrieren sollte.

Die externen Möglichkeiten, das BVerfG von dieser schiefen Ebene abzubringen sind indes reichlich begrenzt. ${ }^{156}$ Sicherlich kann man die Verfassung ändern, um bestimmte Dinge klarzustellen. Abgesehen davon bleibt vor allem die Hoffnung, dass Vernunft und Einsicht obsiegen. In der deutschen Fassung ist der Titel von „Rebel without a cause“" „...denn sie wissen nicht, was sie tun“. ${ }^{157}$ Es ist zu hoffen, dass letzten Endes alle Beteiligten, Richter, Zentralbanker und andere, wissen, was sie tun - und dass sie das Richtige tun.

153 C. Schmitt, Der Hüter der Verfassung, 1931, S. 48.

154 H. Kelsen, Wer soll Hüter der Verfassung sein?, Die Justiz 6 (1931), S. 5.

155 S. hierzu die abweichende Meinung der Richterin Lübbe-Wolff, BVerfG, 2 BvR 2728/13, Beschl. v. 14.1.2014 - OMT, Sondervotum Lübbe-Wolff, Rn. 8.

156 Zum sogenannten ,court-packing“ (Einwirkung auf die Zusammensetzung des Gerichts) als dramatischster Maßnahme und seinen Grenzen s. F. C. Mayer, Kompetenzverschiebungen als Krisenfolge? Die US-Verfassungsentwicklung seit dem New Deal und Lehren für die Euro-Krise, JZ 2014, S. 593.

157 Das vollständige Zitat lautet: „Vater, vergib ihnen; denn sie wissen nicht, was sie tun“, Lukas 23:34. 